\title{
TRIHALOMETHANE AND NONPURGEABLE TOTAL ORGANIC- HALIDE FORMATION POTENTIALS FOR THE MISSISSIPPI RIVER AND SOME OF ITS TRIBUTARIES, MARCH-APRIL 1992
}

By R.E. Rathbun

U.S. Geological Survey

Open-File Report 94-336 


\section{U.S. DEPARTMENT OF THE INTERIOR \\ BRUCE BABBITT, Secretary \\ U.S. GEOLOGICAL SURVEY \\ Gordon P. Eaton, Director}

The use of trade, product, industry, or firm names is for descriptive purposes only and does not imply endorsement by the U.S. Government.

For additional information write to:

Chief, Branch of Regional Research

U.S. Geological Survey

Box 25046, MS 418

Denver Federal Center

Denver, CO 80225
Copies of this report can be purchased from:

U.S. Geological Survey

Earth Science Information Center Open-File Reports Section

Box 25286, MS 517

Denver Federal Center

Denver, CO 80225 


\section{CONTENTS}

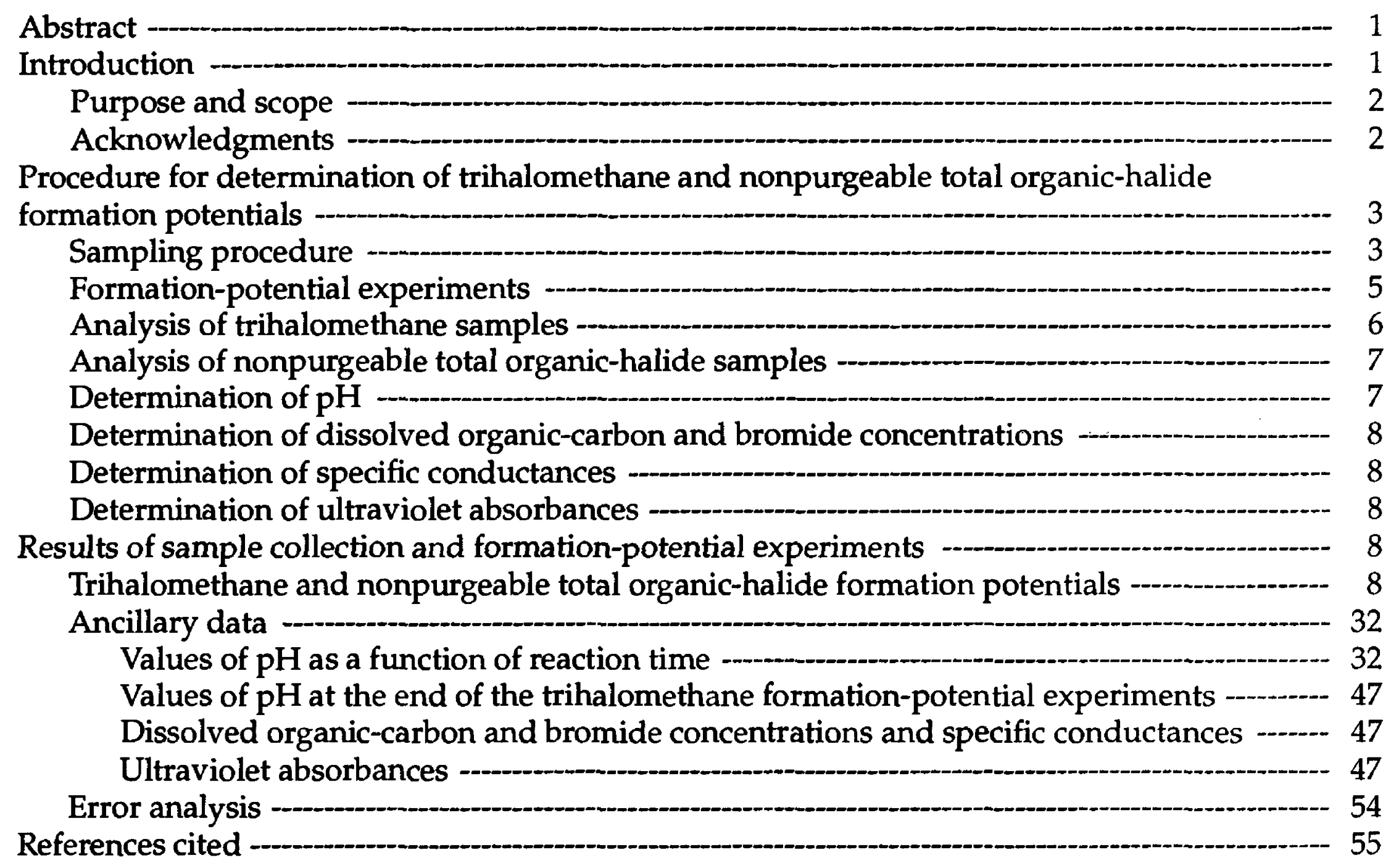

\section{FIGURES}

1. Map showing location of sampling sites in the Mississippi River basin-

2. Graph showing trihalomethane, nonpurgeable total organic-halide, and dissolvedorganic carbon concentrations as a function of cumulative river kilometers upstream from Head of Passes, La.; initial pH of 10.00 and initial free-chlorine concentration of 30.0 milligrams per liter

3. Graph showing trihalomethane and nonpurgeable total organic-halide concentrations for several sampling sites along the Mississippi River as a function of reaction time; initial $\mathrm{pH}$ of 7.50 and initial free-chlorine concentration of 30.0 milligrams per liter- 


\section{TABLES}

Page

1. General location and cumulative river kilometers upstream from Head of Passes, La., for the sampling sites, dates and times of sampling, and water discharges-------- 3

2-15. Concentrations of the four trihalomethane compounds and the total trihalomethane and nonpurgeable total organic-halide concentrations for a reaction time of 168.0 hours as a function of initial $\mathrm{pH}$ and initial free-chlorine concentration:

2. Mississippi River at Minneapolis, Minn., April 6, 1992---12

3. Mississippi River at LaCrosse, Wis., April 3, 1992-..13

4. Mississippi River at Dubuque, Iowa, April 2, 1992 -

5. Mississippi River at Davenport, Iowa, April 2, 1992-

6. Mississippi River at Quincy, Ill., April 1, 1992--..-16

7. Missouri River 1.6 kilometers upstream from confluence with Mississippi River, March 31, 1992--_on-17

8. Mississippi River at St. Louis, Mo., March 31, 1992

9. Mississippi River at Cairo, Ill., March 30, 1992-_...-19

10. Ohio River 1.6 kilometers upstream from confluence with Mississippi River, March 30, 1992--20

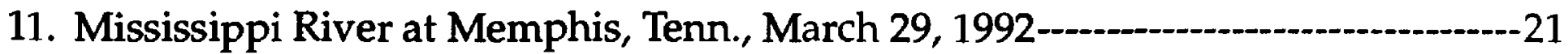

12. Mississippi River at Greenville, Miss., March 28, 1992-.........-22

13. Mississippi River at Natchez, Miss., March 26, 1992-_-_-23

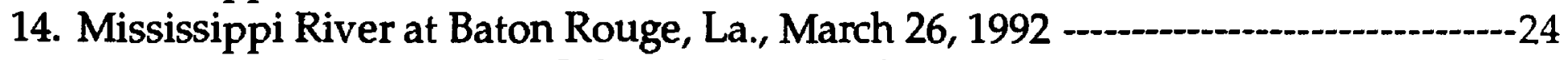

15. Mississippi River at New Orleans, La., March 25, $1992-$

16-27. Concentrations of the four trihalomethane compounds and the total trihalomethane and nonpurgeable total organic-halide concentrations as a function of reaction time for an initial $\mathrm{pH}$ of 7.50 and an initial free-chlorine concentration of 30.0 milligrams per liter:

16. Mississippi River at LaCrosse, Wis., April 3, 1992-

17. Mississippi River at Dubuque, Iowa, April 2, 1992 -

18. Mississippi River at Quincy, Ill., April 1, 1992-_. 27

19. Missouri River 1.6 kilometers upstream from confluence with Mississippi River, March 31, 1992-_-...-27

20. Mississippi River at St. Louis, Mo., March 31, $1992-$

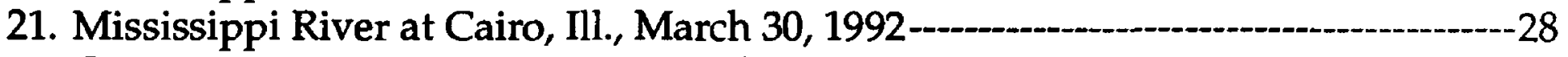

22. Ohio River 1.6 kilometers upstream from confluence with Mississippi River,

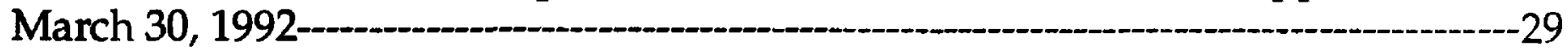

23. Mississippi River at Memphis, Tenn., March 29, 1992--_-29

24. Mississippi River at Greenville, Miss., March 28, $1992-\ldots$

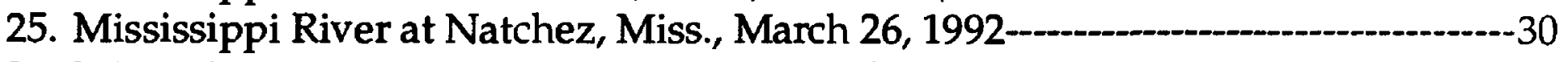

26. Mississippi River at Baton Rouge, La., March 26, 1992 -_-_-31

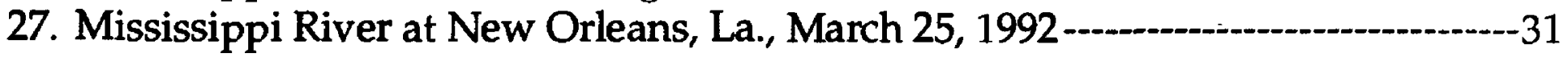


28-41. Variation of $\mathrm{pH}$ with reaction time for different combinations of initial $\mathrm{pH}$ and initial free-chlorine concentration:

28. Mississippi River at Minneapolis, Minn., April 6, $1992-$

29. Mississippi River at LaCrosse, Wis., April 3, 1992-_-33

30. Mississippi River at Dubuque, Iowa, April 2, 1992 -

31. Mississippi River at Davenport, Iowa, April 2, 1992----35

32. Mississippi River at Quincy, Ill., April 1, 1992---

33. Missouri River 1.6 kilometers upstream from confluence with Mississippi River,

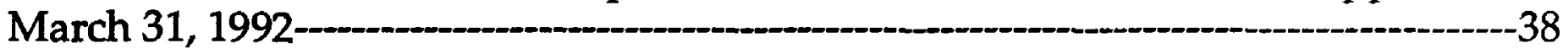

34. Mississippi River at St. Louis, Mo., March 31, 1992-_-_-39

35. Mississippi River at Cairo, Ill., March 30, 1992-_-_._-

36. Ohio River 1.6 kilometers upstream from confluence with Mississippi River, March 30, 1992-

37. Mississippi River at Memphis, Tenn., March 29, 1992--.--42

38. Mississippi River at Greenville, Miss., March 28, 1992-_-_-_4

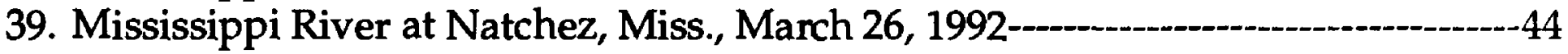

40. Mississippi River at Baton Rouge, La., March 26, 1992 --_.--45

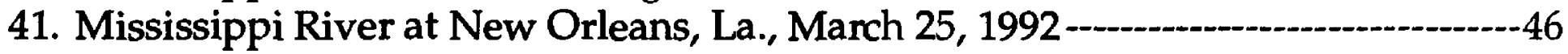

42. Values of $\mathrm{pH}$ at the end of the trihalomethane formation-potential experiments --- 48

43. Dissolved organic-carbon and bromide concentrations and specific conductances of the water samples--

44. Ultraviolet absorbances of the water samples ---

45. Minimum, maximum, and mean values of the coefficient of variation for analysis

of duplicate trihalomethane and nonpurgeable total organic-halide samples -.-.---54 


\section{CONVERSION FACTORS}

\begin{tabular}{lll} 
Multiply & By & To obtain \\
nanometer $(\mathrm{nm})$ & $3.937 \times 10^{-8}$ & inch \\
micrometer $(\mu \mathrm{m})$ & $3.937 \times 10^{-5}$ & inch \\
millimeter $(\mathrm{mm})$ & $3.937 \times 10^{-2}$ & inch \\
meter $(\mathrm{m})$ & $3.937 \times 10^{1}$ & inch \\
kilometer $(\mathrm{km})$ & 0.6214 & mile \\
microgram per liter $(\mu \mathrm{g} / \mathrm{L})$ & $6.243 \times 10^{-8}$ & pound per cubic foot \\
milligram per liter $(\mathrm{mg} / \mathrm{L})$ & $6.243 \times 10^{-5}$ & pound per cubic foot \\
microliter $(\mu \mathrm{L})$ & $3.531 \times 10^{-8}$ & cubic foot \\
milliliter $(\mathrm{mL})$ & $3.531 \times 10^{-5}$ & cubic foot \\
liter $(\mathrm{L})$ & $3.531 \times 10^{-2}$ & cubic foot \\
cubic meter per second $\left(\mathrm{m}^{3} / \mathrm{s}\right)$ & $3.531 \times 10^{1}$ & cubic foot per second \\
\hline
\end{tabular}

Temperature in degree Celsius $\left({ }^{\circ} \mathrm{C}\right)$ may be converted to degree Fahrenheit $\left({ }^{\circ} \mathrm{F}\right)$ using:

$$
{ }^{\circ} \mathrm{F}={ }^{\circ} \mathrm{C}(1.8)+32.0
$$




\title{
TRIHALOMETHANE AND NONPURGEABLE TOTAL ORGANIC- HALIDE FORMATION POTENTIALS FOR THE MISSISSIPPI RIVER AND SOME OF ITS TRIBUTARIES, MARCH-APRIL 1992
}

\author{
R.E. Rathbun
}

\begin{abstract}
Trihalomethane and nonpurgeable total organic-halide formation potentials were determined for the Mississippi River and some of its tributaries from water samples collected between March 25 and April 6, 1992. Formation potentials were measured for 12 sites along the Mississippi River between Minneapolis, Minnesota, and New Orleans, Louisiana, and for the Missouri and Ohio Rivers at sites 1.6 kilometers upstream from their confluences with the Mississippi River. Formation potentials were determined as a function of initial $\mathrm{pH}$ and initial free-chlorine concentration for one reaction time of 168.0 hours at a temperature of 25 degrees Celsius. The formation potentials also were determined as a function of reaction time over a 168.0-hour period for an initial $\mathrm{pH}$ of 7.50 and an initial free-chlorine concentration of 30.0 milligrams per liter. Ancillary measurements include determinations of $\mathrm{pH}$ as a function of reaction time, $\mathrm{pH}$ values at the end of the formation-potential experiments, dissolved organiccarbon and bromide concentrations, ultraviolet absorbances, and specific conductances. All experiments except the reaction-time experiments were done in duplicate, thus permitting an analysis of the errors involved in determining the trihalomethane and nonpurgeable total organichalide formation potentials. Results of the formation-potential determinations, the ancillary measurements, and the error analysis are listed in tables.
\end{abstract}

\section{INTRODUCTION}

The Mississippi River begins in Lake Itasca in north-central Minnesota and drains into the Gulf of Mexico, 3,720 km downstream at a point in Louisiana called Head of Passes. The river flows through Minnesota and Louisiana and forms parts of the borders of Wisconsin, lowa; Illinois, Missouri, Kentucky, Tennessee, Arkansas, and Mississippi. Numerous cities, towns, and villages in these States use the Mississippi River as a source of drinking water. Other population centers along the river might begin to use the Mississippi River as a source of drinking water as ground-water supplies are depleted and as the population increases.

To make water from the Mississippi River safe for drinking, the water first must be disinfected, usually with free chlorine, to eliminate the possibility of the transmission of water-borne diseases such as typhoid fever. Research (Rook, 1974; Bellar and others, 1974; Fleischacker and Randtke, 1983; and Johnson and Jensen, 1986) has indicated, however, that chlorination of natural waters results in the formation of various byproducts, some of which may be health hazards. Therefore, the potential for the formation of these byproducts must be considered when the Mississippi River is used as a source of drinking water.

These byproduct compounds are divided into two classes. The first class consists of the trihalomethane (THM) compounds, which are chlorinated and brominated derivatives of methane. The four compounds commonly included in this class are chloroform, bromodichloromethane, 
chlorodibromomethane, and bromoform. These compounds are volatile and can be determined by gas chromatography techniques. The second class consists of chlorinated and brominated compounds with molecular weights higher than those of the THM compounds. Compounds in this second class are more polar and generally are nonvolatile. They are more difficult to determine as individual compounds; consequently, they commonly are determined together as a bulk parameter called the total organic-halide (TOX) concentration. Because this measurement also includes the THM compounds, the samples usually are purged with an inert gas before analysis to remove the volatile THM compounds. This procedure results in the nonpurgeable total organic-halide (NPTOX) concentration.

The U.S. Geological Survey Mississippi River project is a multidisciplinary study of the waterquality characteristics of the river. Specific topics of study include the distribution of agricultural pesticides and herbicides, trace metals, and industrial organic chemicals among the water, sediment, and biotic phases of the river system and how these substances are transported by the river. Also of interest are the water-quality characteristics of the Mississippi River related to its use as a source of drinking water. Water characteristics affecting this use include constituents already present in the water as well as constituents that are formed when the water is chlorinated to make it safe for drinking. The objective of this report is to describe the results of the determination of disinfection byproducts that could be formed when water from the Mississippi River is chlorinated.

\section{Purpose and Scope}

This report presents the results of the determination of THM and NPTOX formation potentials for the Mississippi River and some of its tributaries. Formation potentials for the THM and NPTOX compounds are defined as the concentrations of these compounds that are formed when water samples are chlorinated at specific conditions of $\mathrm{pH}$, free-chlorine concentration, reaction time, and temperature. In this study, formation potentials were determined for water from 12 sites along the Mississippi River between Minneapolis, Minn., and New Orleans, La., and for water from the Missouri and Ohio Rivers at sites $1.6 \mathrm{~km}$ upstream from their confluences with the Mississippi River. Water samples were collected between March 25, 1992, and April 6, 1992. Formation potentials were determined as a function of initial $\mathrm{pH}$ and initial free-chlorine concentration for one reaction time of 168.0 hours at a temperature of $25^{\circ} \mathrm{C}$. Also, the formation potentials were determined as a function of reaction time over a 168.0-hour period for an initial $\mathrm{pH}$ of 7.50 and an initial free-chlorine concentration of $30.0 \mathrm{mg} / \mathrm{L}$.

\section{Acknowledgments}

Water samples upon which this work was based were collected as a part of the U.S. Geological Survey Mississippi River project under the direction of R.H. Meade. J.A. Moody provided logistical support. Wayne Simoneaux and Bob Cutting piloted the research vessel ACADIANA, and Jean Hough was the cook. L.B. Barber, II, W.L. Campbell, Lesly Conaway, Don Kelly, T.I. Noyes, John Sullivan, and Charles Tabor assisted with the sampling. J.A. Moody and G.S. Ellis collected the Minneapolis, Minn., sample. Appreciation is expressed to all who helped with this study. 


\section{PROCEDURE FOR DETERMINATION OF TRIHALOMETHANE AND NONPURGEABLE TOTAL ORGANIC-HALIDE FORMATION POTENTIALS}

\section{Sampling Procedure}

The 17-m research vessel ACADIANA, owned and operated by the Louisiana Universities Marine Consortium, was used for collecting samples. Grab samples of water were collected by dropping a stainless-steel bucket off the port side of the vessel. The bucket was rinsed once with river water before collecting the sample. Single samples usually were collected from the midpoint of the navigation channel. One exception was the Ohio River, where there was a question about whether the various tributaries flowing into the Ohio River would be mixed at the sampling location. Consequently, a composite sample of equal volumes was collected from points located at 0.1 , 0.5 , and 0.9 of the width measured from the left edge of the water.

On the Mississippi River, the sampling locations generally were slightly upstream from metropolitan areas. General locations in terms of the metropolitan area and specific locations in terms of cumulative river kilometers upstream from Head of Passes, La., for the sampling sites, dates and times of sample collection, and water discharges at the sampling times are listed in table 1. Location of the sampling sites is shown in figure 1.

Table 1.--General location and cumulative river kilometers upstream from Head of Passes, La., for the sampling sites, dates and times of sampling, and water discharges

$\left[\mathrm{m}^{3} / \mathrm{s}\right.$, cubic meters per second]

\begin{tabular}{lcccc}
\hline General location & $\begin{array}{c}\text { Cumulative } \\
\text { river } \\
\text { kilometers }\end{array}$ & Date & $\begin{array}{c}\text { Time } \\
\text { (hours) }\end{array}$ & $\begin{array}{c}\text { Water } \\
\text { discharge } \\
\left.\text { (m } \mathbf{m}^{\mathbf{3}} \mathbf{s}\right)\end{array}$ \\
\hline Minneapolis, Minn. & $2,915.2$ & $04-06-92$ & 1030 & 310 \\
LaCrosse, Wis. & $2,666.9$ & $04-03-92$ & 1229 & 1,900 \\
Dubuque, lowa & $2,470.7$ & $04-02-92$ & 2046 & 2,700 \\
Davenport, Iowa & $2,324.6$ & $04-02-92$ & 1031 & 2,600 \\
Quincy, Ill. & $2,068.4$ & $04-01-92$ & 1241 & 3,600 \\
Missouri River & $1,850.8$ & $03-31-92$ & 1554 & 2,100 \\
St. Louis, Mo. & $1,825.1$ & $03-31-92$ & 1403 & 7,800 \\
Cairo, Ill. & $1,553.6$ & $03-30-92$ & 1422 & 8,000 \\
Ohio River & $1,536.5$ & $03-30-92$ & 1252 & 8,900 \\
Memphis, Tenn. & $1,194.1$ & $03-29-92$ & 0640 & 23,100 \\
Greenville, Miss. & 877.1 & $03-28-92$ & 0009 & 26,400 \\
Natchez, Miss. & 597.4 & $03-26-92$ & 2252 & 25,300 \\
Baton Rouge, La. & 386.7 & $03-26-92$ & 0536 & 22,200 \\
New Orleans, La. & 169.3 & $03-25-92$ & 0906 & 22,300 \\
\hline
\end{tabular}

${ }^{1}$ Discharge data from Moody (in press).

${ }^{2}$ Sampling site 1.6 kilometers upstream from confluence with Mississippi River. 


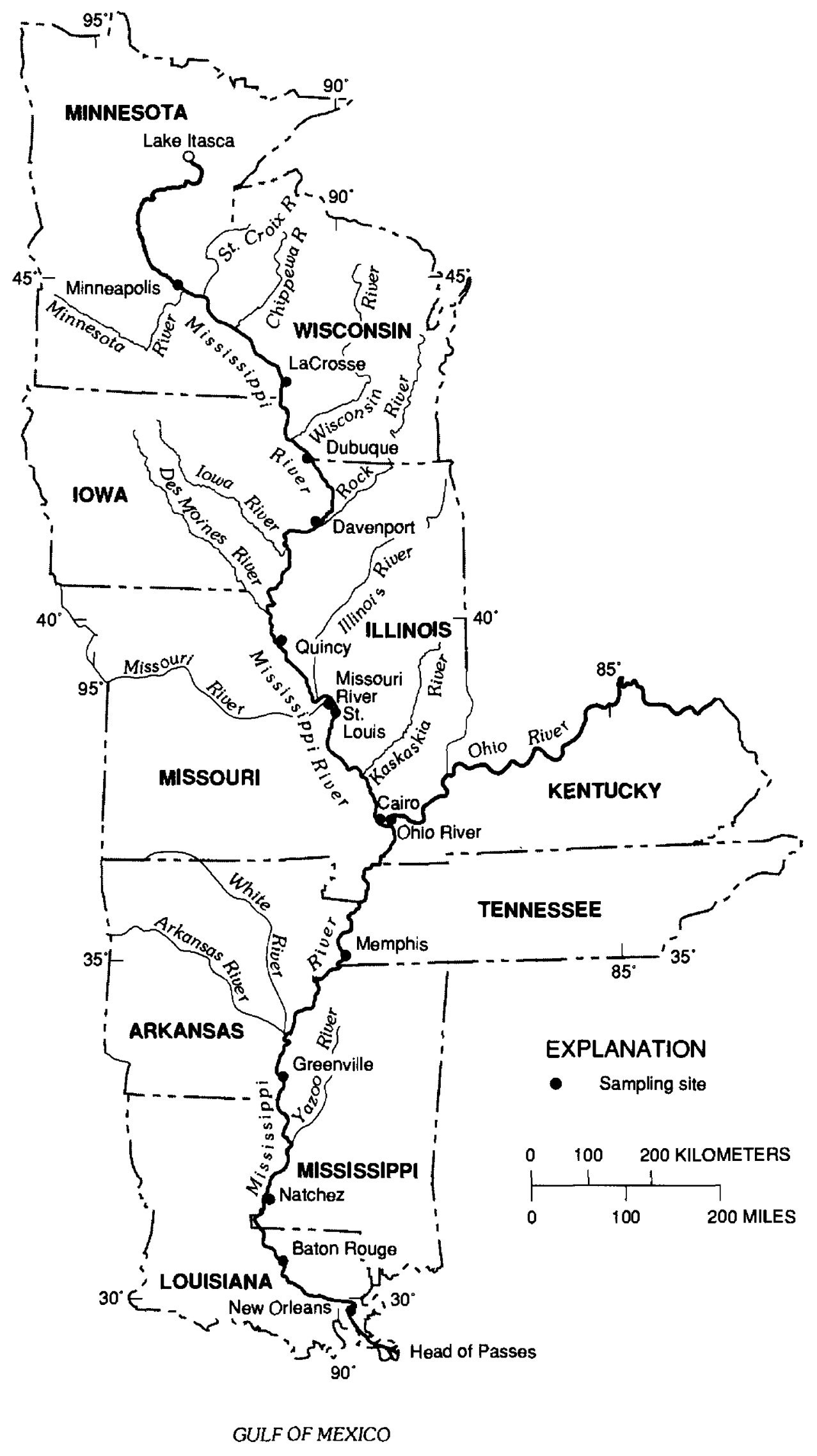

Figure 1.--Location of sampling sites in the Mississippi River basin. 
The water sample collected at each site was transferred from the bucket to a stainless-steel reservoir for pressure filtration. The water was filtered through low-extractable $0.45-\mu \mathrm{m}$ membrane filters that were prerinsed with about $2 \mathrm{~L}$ of high-purity water from a Barnstead Nanopure water system. The first $600 \mathrm{~mL}$ of sample water through the filter was discarded. The water collected at each site was filtered into six 1-L amber glass bottles with Teflon-lined caps. The sample bottles were chilled with ice until shipment to the laboratory in Arvada, Colo. Shipment was by overnight air freight in insulated coolers containing refrigerant cold packs. Water samples in the laboratory were stored at $4^{\circ} \mathrm{C}$ until the formation-potential experiments were done.

\section{Formation-Potential Experiments}

The formation potentials for the THM and NPTOX compounds were determined as a function of initial $\mathrm{pH}$ and initial free-chlorine concentration at $25^{\circ} \mathrm{C}$ for a reaction time of 168.0 hours. Initial $\mathrm{pH}$ values of $5.50,7.50$, and 10.00 and initial free-chlorine concentrations of $15.0,30.0$, and $50.0 \mathrm{mg} / \mathrm{L}$ were used, giving nine THM and nine NPTOX formation potentials for each water sample. These large initial free-chlorine concentrations were used to satisfy any initial chlorine

demand of the water sample and to ensure a residual free-chlorine concentration at the end of the 168.0-hour reaction time. The 168.0-hour reaction time was used to cover the maximum possible time water might be in a distribution system before use. Formation potentials also were determined as a function of time during the 168.0-hour period for an initial $\mathrm{pH}$ of 7.50 and an initial free-chlorine concentration of $30.0 \mathrm{mg} / \mathrm{L}$.

The formation-potential experiments were done in 40-mL amber glass vials with Teflon-faced septum caps. Initiation of an experiment consisted of adjusting the $\mathrm{pH}$ of the water sample to the desired initial $\mathrm{pH}$ using 0.1 normal and 1.0 normal sulfuric acid and sodium hydroxide. The desired initial free-chlorine concentration was obtained by adding the appropriate volume of a reagent-grade sodium hypochlorite solution. The free-chlorine concentration of this solution was determined prior to the start of each experiment by using an amperometric titration procedure. Blank samples of each water sample were taken before the addition of the sodium hypochlorite solution.

Sample vials were filled completely so that no head space existed. Vials were placed in an incubator at $25^{\circ} \mathrm{C}$. All 168.0-hour experiments were done with duplicate vials. At the end of 168.0 hours, the experiments were terminated by quenching the remaining free chlorine with a sodium thiosulfate solution for the THM experiments and sodium sulfite crystals for the NPTOX experiments. For the reaction-time experiments, samples were quenched at approximate times of $0,2,6,12,24,48,72,120$, and 168.0 hours.

Before quenching the NPTOX samples, $5.0 \mathrm{~mL}$ of sample was removed from each of the vials and used to estimate the residual free-chlorine concentrations. Because a precise determination of the residual free-chlorine concentration was not needed, a Hach chlorine test kit with a range from 0 to $3.5 \mathrm{mg} / \mathrm{L}$ with $0.1-\mathrm{mg} / \mathrm{L}$ divisions was used for this purpose. After acidification to a $\mathrm{pH}$ of about 2 with nitric acid, the NPTOX samples were purged for 10 minutes with ultrahigh-purity nitrogen gas to remove the THM compounds. Samples were stored in an incubator at $4^{\circ} \mathrm{C}$ until analysis. 


\section{Analysis of Trihaiomethane Samples}

Concentrations of the THM compounds in the samples were determined using a solventextraction procedure (Federal Register, 1979) with pentane as the solvent. The procedure was modified slightly in that the sample extractions were done directly in the sample vials to avoid having to transfer a sample containing volatile constituents. The procedure consisted of removing $4.5 \mathrm{~mL}$ of sample with an adjustable pipetter and discarding, adding $4.0 \mathrm{~mL}$ of pentane with a Class A pipet, and shaking the mixture for 1.5 minutes The precise amounts of sample removed and pentane added were determined by weighing the vials after each step. The Burdick and Jackson brand of pentane especially formulated for THM analyses was used. The vials containing the water and pentane layers were stored upside down at $4^{\circ} \mathrm{C}$ until analysis. Storing the samples upside down precluded any loss of the pentane or the THM compounds from the sample vial during storage.

The pentane extracts were analyzed with a Hewlett-Packard 5880-A gas chromatograph equipped with an electron-capture detector. A $12-\mathrm{m}$ long by $0.2-\mathrm{mm}$ inside diameter HewlettPackard, cross-linked, methyl silicone-gum capillary column with a $0.33 \mu \mathrm{m}$ film thickness was used with the purged splitless technique. Retention times on this column were $0.92,1.42,2.49$, and 4.77 minutes for chloroform, bromodichloromethane, chlorodibromomethane, and bromoform, respectively.

Volumes of the $40-\mathrm{mL}$ sample vials were not precisely $40.0 \mathrm{~mL}$ and were not consistent. To adjust for variations in the vial volumes, the vials were weighed at each step of the extraction process, beginning with the empty vial and ending with the final sample/pentane step. These weights permitted determination of the exact water/pentane volume ratio for each sample. The peak areas for each sample were adjusted from the volume ratio of that sample to the average volume ratio for the standard samples for that particular run. This procedure resulted in all samples and standards being adjusted to a single water/pentane ratio.

Standard samples of known concentrations of each of the four THM compounds were prepared according to an established procedure (Federal Register, 1979). The procedure consists of adding each of the four THM compounds to methanol in a 10-mL volumetric flask. The amount added was determined approximately by volume and precisely by weighing on a five-place analytical balance. Two additional primary standards were prepared by serial dilution of this standard using gas-tight syringes. Secondary standards covering the expected range of sample concentrations were prepared by injecting microliter quantities of the primary standards into river water contained in 40-mL septum vials. River water was used to prepare the standards to eliminate any possible effect of the water chemistry on the efficiency of the extraction procedure. These secondary standards were treated exactly as the samples from the experiments except that sodium hypochlorite was not added to the standards. A new set of standards was prepared for each river-water sample.

A large number of standards was necessary because the response of the electron-capture detector was somewhat nonlinear and the standards had to cover a wide range of concentrations so that the peak areas of each of the four THM compounds in every sample were bracketed by the peak areas of the standards. The number of standards ranged from 14 for the Minneapolis sample to 10 for the New Orleans sample. These standards were analyzed twice each day, resulting in 44 to 60 percent of the samples analyzed each day being standards. 


\section{Analysis of Nonpurgeable Total Organic-Halide Samples}

Concentrations of the NPTOX compounds in the samples were determined using a Dohrmann DX-20A TOX analyzer with an AD-3 adsorption module. This procedure involved adsorption of the organic compounds from the water sample onto granular activated carbon packed in glass columns. Two of these columns were used in series for each sample. Following adsorption of the sample, the contents of the columns were washed with $2 \mathrm{~mL}$ of a 0.08 normal potassium nitrate solution to remove inorganic halides. The granular activated carbon then was removed from the columns with a push rod into the quartz sample boat of the instrument. The analysis was started by moving the sample boat into the furnace at $800^{\circ} \mathrm{C}$ where the chlorinated and brominated compounds were volatilized and converted in the presence of oxygen to an equivalent amount of hydrogen halides. These ions were titrated in a microcoulometric cell, resulting in the determination of the mass of NPTOX compounds in the original sample. The NPTOX concentration was computed from this mass and the volume of sample sorbed in the adsorption step.

The high concentrations of NPTOX compounds resulting from the formation-potential experiments required dilution of the samples to assure that the concentrations were within the response range of the instrument. Samples were diluted with high-purity water from a Barnstead Nanopure system. A blank for this dilution water was analyzed each day samples were run. Usually, a $30-\mathrm{mL}$ mixture was prepared, with the sample amount ranging from 5 to $25 \mathrm{~mL}$, depending on the expected concentration. Ultrahigh-purity nitrogen gas was used to force $25 \mathrm{~mL}$ of this 30-mL sample through the glass columns containing the granular activated carbon. All aliquots were measured with Class A pipets.

Operation of the microcoulometric cell was verified at the beginning of each day by injections of a sodium chloride standard directly into the electrolyte of the cell. These verifications also were done any time during the day when it became necessary to change the cell electrolyte. Recovery of the instrument was checked at the beginning of each day by injections of a trichlorophenol standard onto granular activated-carbon ash in the instrument boat and subsequent analysis of the trichlorophenol standard. These recovery checks also were repeated any time during the day when it became necessary to change the cell electrolyte and on any other occasion when operation of the instrument might be questionable. If results of these checks deviated by more than 10 percent from the expected values, steps were taken to improve the performance of the instrument by changing the cell electrolyte and the inlet and exit tube liners and by cleaning the hatch area.

\section{Determination of $\mathrm{pH}$}

The $\mathrm{pH}$ values of the natural water samples ranged from about 7.8 to about 8.2 . For the formation potential experiments, the $\mathrm{pH}$ was adjusted to initial $\mathrm{pH}$ values of $5.50,7.50$, and 10.00 . Because these water samples were not buffered, the $\mathrm{pH}$ of the 5.50 and 7.50 water samples increased, and the $\mathrm{pH}$ of the 10.00 water samples decreased slightly when the basic sodium hypochlorite solution was added to provide the free-chlorine concentration. To determine the variation of the $\mathrm{pH}$ with time, the $\mathrm{pH}$ values of each of the nine combinations of initial $\mathrm{pH}$ and initial free-chlorine concentration were determined at reaction times of approximately $0,24,48$, $72,120,144$, and 168 hours.

Measurements were made using an Orion Model $811 \mathrm{pH}$ meter with a 91-02 research-grade $\mathrm{pH}$ probe. These samples were prepared at the same time the vials for the formation-potential experiments were filled. Amber glass bottles having Teflon-lined caps, a volume of $70 \mathrm{~mL}$, and a mouth wide enough to accommodate the $\mathrm{pH}$ and temperature probes of the instrument were used 
for this purpose. The $\mathrm{pH}$ meter was calibrated each day before the measurements were done. A two-point calibration using buffers of 6.86 and 10.00 bracketed all the experimental $\mathrm{pH}$ values.

The $\mathrm{pH}$ values of the THM formation-potential experiments also were determined after completion of the gas chromatographic analyses. The pentane was evaporated, and the $\mathrm{pH}$ of the remaining water was determined for each of the nine samples.

\section{Determination of Dissolved Organic-Carbon and Bromide Concentrations}

Dissolved organic-carbon (DOC) and bromide concentrations of the water samples were determined at the U.S. Geological Survey National Water Quality Laboratory in Arvada, Colo. DOC concentration was determined using the wet-oxidation method (Wershaw and others, 1987). Bromide concentrations were determined using segmented-flow automated colorimetry (Fishman and Friedman, 1989).

\section{Determination of Specific Conductances}

Specific conductances of the sample waters were measured using a Yellow Springs Instrument Company model 32 conductance meter. Temperatures of the water samples were adjusted to $25.0^{\circ} \mathrm{C}$ so that no temperature corrections were necessary.

\section{Determination of Ultraviolet Absorbances}

The ultraviolet (UV) absorbance of natural waters has been used as a predictor of the DOC concentration. Various UV wavelengths have been used (Buffle and others, 1978; Oliver and Thurman, 1981; Edzwald and others, 1985; Ceraso, 1987; Chadick and Amy, 1987; Moore, 1987). UV absorbances of the water samples were determined at wavelengths of $254,280,330$, and 400 $\mathrm{nm}$. A Spectronics model 2000 spectrophotometer with a $50-\mathrm{mm}$ cell was used for these determinations. Absorbances were measured for both the $\mathrm{pH}$-adjusted water samples and the natural water samples.

\section{RESULTS OF SAMPLE COLLECTION AND FORMATION-POTENTIAL EXPERIMENTS}

\section{Trihalomethane and Nonpurgeable Total Organic-Halide Formation Potentiais}

The potentials for formation of THM and NPTOX compounds when the water samples were treated with free chlorine are expressed as the concentrations of these compounds formed in the experiments. Two types of results were obtained. The first type consisted of concentrations of the THM and NPTOX compounds formed during a reaction time of 168.0 hours for nine combinations of initial $\mathrm{pH}$ and initial free-chlorine concentration. The second type consisted of concentrations of the THM and NPTOX compounds formed as a function of time during the reaction time of 168.0 hours for an initial $\mathrm{pH}$ of 7.50 and an initial free-chlorine concentration of $30.0 \mathrm{mg} / \mathrm{L}$. Typical results of the first type (fig. 2) show THM and NPTOX concentrations for an initial pH of 10.00 and an initial free-chlorine concentration of $30.0 \mathrm{mg} / \mathrm{L}$ as a function of cumulative river kilometers upstream from Head of Passes, La. The DOC concentrations also are shown in figure 2. Typical results of the second type (fig. 3) show THM and NPTOX concentrations for several sampling sites along the Mississippi River as a function of reaction time. 


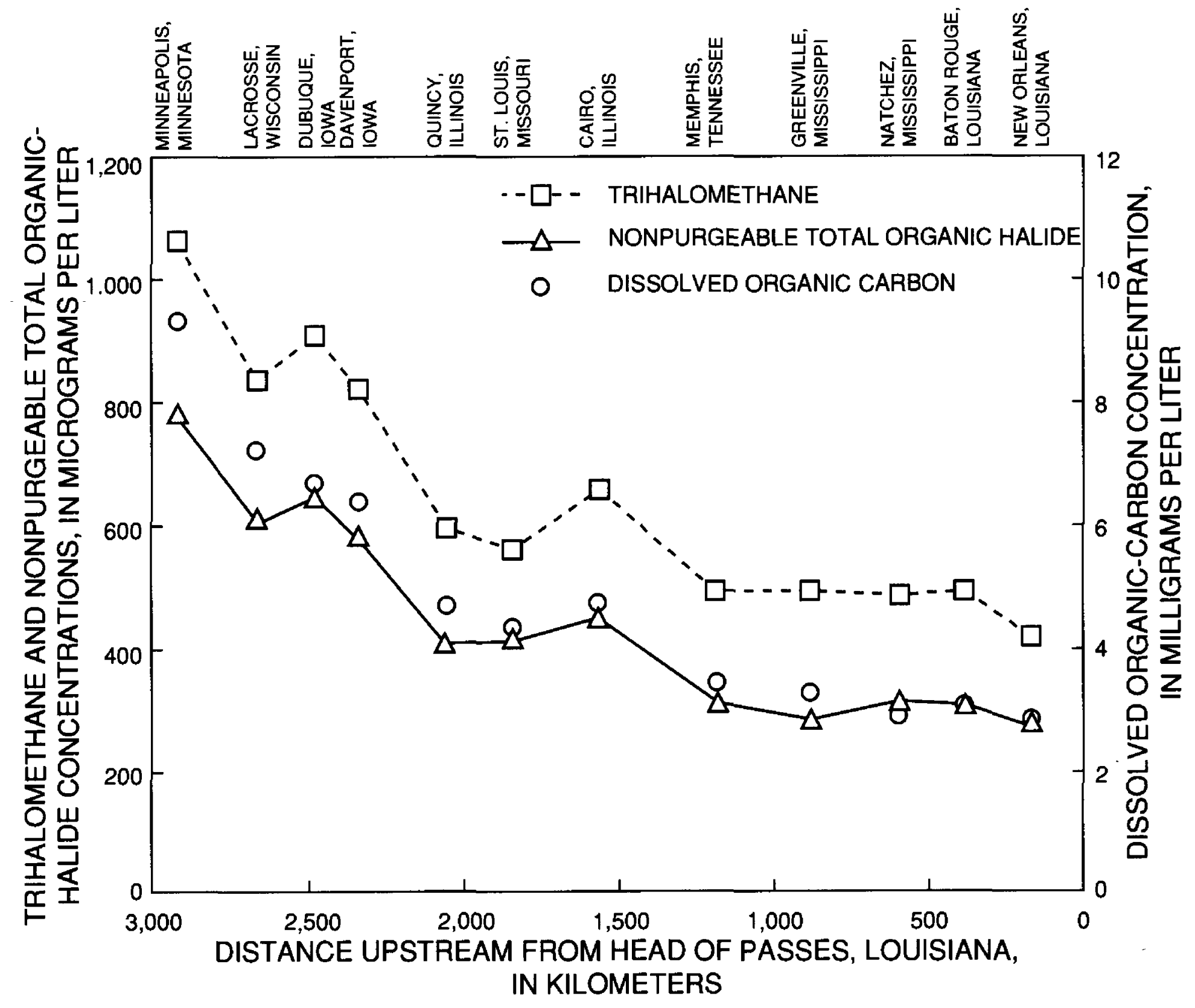

Figure 2.--Trihalomethane, nonpurgeable total organic-halide, and dissolved organiccarbon concentrations as a function of cumulative river kilometers upstream from Head of Passes, La.; initial pH of $\mathbf{1 0 . 0 0}$ and initial free-chlorine concentration of $\mathbf{3 0 . 0}$ milligrams per liter. 

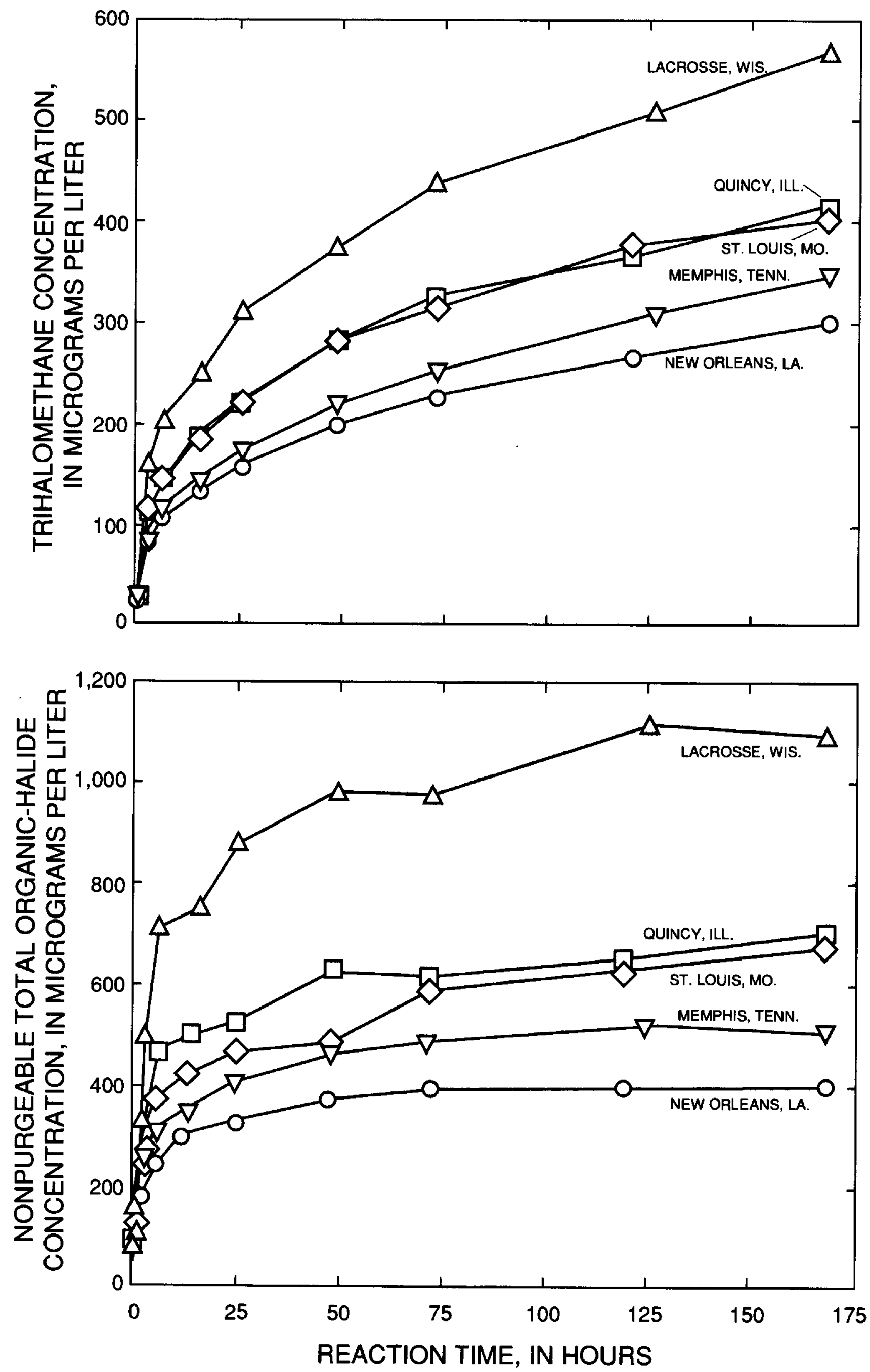

Figure 3.--Trihalomethane and nonpurgeable total organic-halide concentrations as a function of reaction time for several sampling sites along the Mississippi River; initial $\mathrm{pH}$ of 7.50 and initial free-chlorine concentration of 30.0 milligrams per liter. 
All concentrations were corrected for blank contributions. The blank corrections for chloroform ranged from 0.00 to $1.33 \mu \mathrm{g} / \mathrm{L}$ and averaged $0.35 \mu \mathrm{g} / \mathrm{L}$ for 45 determinations. Average blank corrections for the other three THM compounds were less than $0.02 \mu \mathrm{g} / \mathrm{L}$. Blank corrections for the NPTOX concentrations ranged from 5.08 to $30.6 \mu \mathrm{g} / \mathrm{L}$ and averaged $14.5 \mu \mathrm{g} / \mathrm{L}$ for 39 determinations for samples from the Mississippi River. Corrections for the Missouri River water sample ranged from 11.1 to $13.7 \mu \mathrm{g} / \mathrm{L}$ and averaged $12.7 \mu \mathrm{g} / \mathrm{L}$. Corrections for the Ohio River water sample ranged from 11.0 to $12.2 \mu \mathrm{g} / \mathrm{L}$ and averaged $11.4 \mu \mathrm{g} / \mathrm{L}$.

The three chlorinated THM compounds (chloroform, bromodichloromethane, and chlorodibromomethane) were detected in all samples. Bromoform was not detected in 49 percent of the samples. The detection limit for bromoform for the analytical procedure used in this study was estimated to be about $0.01 \mu \mathrm{g} / \mathrm{L}$.

For the first type of results, duplicate experiments were done for all nine combinations of initial $\mathrm{pH}$ and initial free-chlorine concentration for each water sample. The two vials for the Minneapolis sample for an initial $\mathrm{pH}$ of 7.50 and an initial free-chlorine concentration of $15 \mathrm{mg} / \mathrm{L}$ had no detectable free-chlorine concentration at the end of the reaction time. The limit of detection of the procedure used for these free-chlorine measurements was $0.1 \mathrm{mg} / \mathrm{L}$. Concentrations for these samples are flagged with an asterisk in tables 2 to indicate that these concentrations could have been affected by the lack of a free-chlorine residual at the end of the experiments.

For the second type of results, duplicate experiments were done for a reaction time of 168.0 hours and single experiments were done for all other reaction times. The 0.00 -hour reaction-time samples were samples taken and quenched immediately after addition of the sodium hypochlorite solution to the water sample. The time required to add the sodium hypochlorite solution to the water sample in a separatory funnel, mix the contents, fill the 0.00 -hour time vials already containing the quenching agent, and to shake these vials ranged from 30 to 45 seconds.

The first type of results consisting of concentrations of the four THM compounds and the total THM and NPTOX concentrations for a reaction time of 168.0 hours and nine combinations of initial $\mathrm{pH}$ and free-chlorine concentration are listed in tables 2-15. The second type of results consisting of concentrations of the four THM compounds and the total THM and NPTOX concentrations as a function of reaction time for an initial $\mathrm{pH}$ of 7.50 and an initial free-chlorine concentration of $30.0 \mathrm{mg} / \mathrm{L}$ are listed in tables 16-27. The concentrations for the 168.0-hour reaction time for an initial $\mathrm{pH}$ of 7.50 and an initial free-chlorine concentration of $30.0 \mathrm{mg} / \mathrm{L}$ are included in both the first and the second types of results presented in tables 2-15 and in tables 16-27.

One bottle of the Minneapolis water sample and three bottles of the Davenport water sample froze during storage. Most of the Minneapolis water sample was lost, but almost all of the Davenport water sample was recovered by melting the ice. Previous unpublished results indicated that the THM formation potential of thawed water was considerably less than that of water that had not been frozen. Therefore, formation potential experiments for the Davenport water sample were done for both the thawed water sample and the water sample that had not been frozen. Because of the limited amount of water, however, formation-potential experiments could be done for only six of the nine combinations of initial $\mathrm{pH}$ and initial free-chlorine concentration for the thawed-water sample and seven of the nine combinations for the regular water sample (table 5). Also, the concentration-time formation-potential experiments could not be done for the Minneapolis and Davenport water samples because of insufficient water. 
Table 2.--Concentrations of the four trihalomethane compounds and the total trihalomethane and nonpurgeable total organic-halide concentrations for a reaction time of $\mathbf{1 6 8 . 0}$ hours as a function of initial $\mathrm{pH}$ and initial free-chlorine concentration, Mississippi River at Minneapolis, Minn., April 6, 1992

$[\mu \mathrm{g} / \mathrm{L}$, microgram per liter; $\mathrm{CL}$, free-chlorine concentration; $\mathrm{mg} / \mathrm{L}$, milligram per liter; $\mathrm{CHCl}_{3}$, chloroform; $\mathrm{BrCHCl}_{2}$, bromodichloromethane; $\mathrm{ClCHBr}_{2}$, chlorodibromomethane; $\mathrm{CHBr}_{3}$, bromoform; THM, trihalomethane; NPTOX, nonpurgeable total organic halide; ND, not detected]

\begin{tabular}{|c|c|c|c|c|c|c|c|}
\hline \multirow{2}{*}{ initial pH } & \multirow{2}{*}{$\begin{array}{c}\text { Initial CL } \\
\text { (mg/L) }\end{array}$} & \multicolumn{6}{|c|}{$\begin{array}{c}\text { Concentration } \\
(\mu g / L)\end{array}$} \\
\hline & & $\mathrm{CHCl}_{3}$ & $\mathrm{BrCHCl}_{2}$ & $\mathrm{ClCHBr}_{2}$ & $\mathrm{CHBr}_{3}$ & $\begin{array}{l}\text { Total } \\
\text { THM }\end{array}$ & NPTOX \\
\hline 5.50 & 15.0 & 384 & 15.6 & 0.25 & $\overline{N D}$ & 400 & 1,270 \\
\hline 5.50 & 15.0 & 388 & 16.1 & .30 & ND & 404 & 1,310 \\
\hline 5.50 & 30.0 & 531 & 17.2 & .24 & ND & 548 & 1,630 \\
\hline 5.50 & 30.0 & 514 & 16.3 & .27 & ND & 531 & 1,650 \\
\hline 5.50 & 50.0 & 639 & 18.0 & .26 & ND & 657 & 1,770 \\
\hline 5.50 & 50.0 & 637 & 17.6 & .23 & ND & 655 & 1,860 \\
\hline 7.50 & 15.0 & $480^{*}$ & $19.4 *$ & $.69^{*}$ & $\mathrm{ND}^{*}$ & $500 *$ & $852 *$ \\
\hline 7.50 & 15.0 & $475^{*}$ & $19.0 *$ & $.38^{*}$ & $\mathrm{ND}^{*}$ & $494 *$ & $836^{*}$ \\
\hline 7.50 & 30.0 & 779 & 24.7 & .69 & ND & 804 & 1,350 \\
\hline 7.50 & 30.0 & 755 & 22.4 & .55 & ND & 778 & 1,290 \\
\hline 7.50 & 50.0 & 899 & 22.4 & .42 & ND & 922 & 1,370 \\
\hline 7.50 & 50.0 & 903 & 23.9 & .47 & ND & 927 & 1,340 \\
\hline 10.00 & 15.0 & 813 & 18.4 & .36 & ND & 832 & 654 \\
\hline 10.00 & 15.0 & 823 & 18.2 & .40 & ND & 842 & 653 \\
\hline 10.00 & 30.0 & 1,050 & 22.8 & .49 & ND & 1,070 & 798 \\
\hline 10.00 & 30.0 & 1,050 & 21.5 & .43 & ND & 1,070 & 754 \\
\hline 10.00 & 50.0 & 1,190 & 24.1 & .52 & ND & 1,210 & 884 \\
\hline 10.00 & 50.0 & 1,160 & 23.6 & .48 & ND & 1,180 & 955 \\
\hline
\end{tabular}

${ }^{*}$ Free-chlorine concentration less than the detection limit at reaction time of 168.0 hours. 
Table 3.--Concentrations of the four trihalomethane compounds and the total trihalomethane and nonpurgeable total organic-halide concentrations for a reaction time of $\mathbf{1 6 8 . 0}$ hours as a function of initial pH and initial free-chlorine concentration, Mississippi River at LaCrosse, Wis., April 3, 1992

$[\mu \mathrm{g} / \mathrm{L}$, microgram per liter; $\mathrm{CL}$, free-chlorine concentration; $\mathrm{mg} / \mathrm{L}$, milligram per liter; $\mathrm{CHCl}_{3}$, chloroform; $\mathrm{BrCHCl}_{2}$, bromodichloromethane; $\mathrm{ClCHBr}_{2}$, chlorodibromomethane; $\mathrm{CHBr}_{3}$, bromoform; THM, trihalomethane; NPTOX, nonpurgeable total organic halide; ND, not detected]

\begin{tabular}{|c|c|c|c|c|c|c|c|}
\hline \multirow{2}{*}{ Initial pH } & \multirow{2}{*}{$\begin{array}{c}\text { Initial CL } \\
\text { (mg/L) }\end{array}$} & \multicolumn{6}{|c|}{$\begin{array}{c}\text { Concentration } \\
(\mu \mathrm{g} / \mathrm{L})\end{array}$} \\
\hline & & $\mathrm{CHCl}_{3}$ & $\mathrm{BrCHCl}_{2}$ & $\mathrm{ClCHBr}_{2}$ & $\mathrm{CHBr}_{3}$ & $\begin{array}{l}\text { Total } \\
\text { THM }\end{array}$ & NPTOX \\
\hline 5.50 & 15.0 & 338 & 17.5 & 0.48 & $\overline{\mathrm{ND}}$ & 356 & 993 \\
\hline 5.50 & 15.0 & 334 & 18.0 & .49 & ND & 352 & 1,100 \\
\hline 5.50 & 30.0 & 428 & 18.4 & .45 & ND & 447 & 1,250 \\
\hline 5.50 & 30.0 & 424 & 18.3 & .46 & ND & 443 & 1,210 \\
\hline 5.50 & 50.0 & 507 & 19.2 & .46 & ND & 527 & 1,420 \\
\hline 5.50 & 50.0 & 504 & 18.5 & .46 & ND & 523 & 1,500 \\
\hline 7.50 & 15.0 & 493 & 23.9 & .81 & ND & 518 & 947 \\
\hline 7.50 & 15.0 & 479 & 22.4 & .75 & ND & 502 & 909 \\
\hline 7.50 & 30.0 & 536 & 22.8 & .71 & ND & 560 & 1,100 \\
\hline 7.50 & 30.0 & 547 & 24.0 & .79 & ND & 572 & 1,070 \\
\hline 7.50 & 50.0 & 714 & 24.7 & .74 & ND & 739 & 1,180 \\
\hline 7.50 & 50.0 & 731 & 24.8 & .73 & ND & 757 & 1,120 \\
\hline 10.00 & 15.0 & 681 & 23.5 & 1.01 & ND & 706 & 621 \\
\hline 10.00 & 15.0 & 688 & 24.0 & .90 & ND & 713 & 610 \\
\hline 10.00 & 30.0 & 801 & 25.7 & .94 & ND & 828 & 621 \\
\hline 10.00 & 30.0 & 815 & 26.0 & .88 & ND & 842 & 594 \\
\hline 10.00 & 50.0 & 875 & 26.4 & .97 & ND & 902 & 678 \\
\hline 10.00 & 50.0 & 885 & 26.5 & .98 & ND & 912 & 649 \\
\hline
\end{tabular}


Table 4.--Concentrations of the four trihalomethane compounds and the total trihalomethane and nonpurgeable total organic-halide concentrations for a reaction time of 168.0 hours as a function of initial $\mathrm{pH}$ and initial free-chlorine concentration, Mississippi River at Dubuque, lowa, April 2, 1992

[ $\mu \mathrm{g} / \mathrm{L}$, microgram per liter; $\mathrm{CL}$, free-chlorine concentration; $\mathrm{mg} / \mathrm{L}$, milligram per liter; $\mathrm{CHCl}_{3}$, chloroform; $\mathrm{BrCHCl}_{2}$, bromodichloromethane; $\mathrm{ClCHBr}$, chlorodibromomethane; $\mathrm{CHBr}_{3}$, bromoform; THM, trihalomethane; NPTOX, nonpurgeable total organic halide; ND, not detected]

\begin{tabular}{cccccccr}
\hline & & \multicolumn{5}{c}{$\begin{array}{c}\text { Concentration } \\
(\mu \mathbf{g} / \mathbf{L})\end{array}$} \\
\cline { 3 - 8 } Initial pH & $\begin{array}{c}\text { Initial } \\
(\mathbf{m g} / \mathbf{L})\end{array}$ & $\mathrm{CHCl}_{3}$ & $\mathrm{BrCHCl}_{2}$ & $\mathbf{C l C H B r}_{2}$ & $\mathrm{CHBr}_{3}$ & $\begin{array}{r}\text { Total } \\
\text { THM }\end{array}$ & NPTOX \\
\hline 5.50 & 15.0 & 336 & 15.3 & 0.41 & ND & 352 & 970 \\
5.50 & 15.0 & 339 & 15.5 & .42 & ND & 355 & 955 \\
5.50 & 30.0 & 423 & 15.1 & .40 & ND & 438 & 1,160 \\
5.50 & 30.0 & 423 & 14.9 & .38 & ND & 438 & 1,130 \\
5.50 & 50.0 & 531 & 15.7 & .34 & ND & 547 & 1,340 \\
5.50 & 50.0 & 530 & 16.2 & .40 & ND & 547 & 1,250 \\
& & & & & & & \\
7.50 & 15.0 & 469 & 18.7 & .53 & ND & 488 & 797 \\
7.50 & 15.0 & 477 & 19.1 & .54 & ND & 497 & 826 \\
7.50 & 30.0 & 553 & 19.9 & .57 & ND & 573 & 1,020 \\
7.50 & 30.0 & 557 & 20.0 & .55 & ND & 578 & 1,050 \\
7.50 & 50.0 & 712 & 20.9 & .55 & ND & 733 & 1,020 \\
7.50 & 50.0 & 706 & 20.2 & .51 & ND & 727 & 1,090 \\
& & & & & & & \\
10.00 & 15.0 & 726 & 19.6 & .66 & ND & 746 & 586 \\
10.00 & 15.0 & 723 & 19.2 & .65 & ND & 743 & 515 \\
10.00 & 30.0 & 901 & 22.0 & .71 & ND & 924 & 626 \\
10.00 & 30.0 & 888 & 21.3 & .60 & ND & 910 & 659 \\
10.00 & 50.0 & 973 & 22.2 & .62 & ND & 996 & 785 \\
10.00 & 50.0 & 987 & 22.6 & .64 & ND & 1,010 & 737 \\
\hline
\end{tabular}


Table 5.--Concentrations of the four trihalomethane compounds and the total trihalomethane and nonpurgeable total organic-halide concentrations for a reaction time of 168.0 hours as a function of initial pH and inltiai free-chlorine concentration, Mississippi River at Davenport, lowa, April 2, 1992

$[\mu \mathrm{g} / \mathrm{L}$, microgram per liter; $\mathrm{CL}$, free-chlorine concentration; $\mathrm{mg} / \mathrm{L}$, milligram per liter; $\mathrm{CHCl}_{3}$, chloroform; $\mathrm{BrCHCl}_{2}$, bromodichloromethane; $\mathrm{ClCHBr}$, chlorodibromomethane; $\mathrm{CHBr}_{3}$, bromoform; THM, trihalomethane; NPTOX, nonpurgeable total organic halide; ND, not detected]

\begin{tabular}{|c|c|c|c|c|c|c|c|}
\hline \multirow{2}{*}{ Initial pH } & \multirow{2}{*}{$\begin{array}{l}\text { Initlal CL } \\
\text { (mg/L) }\end{array}$} & \multicolumn{6}{|c|}{$\begin{array}{c}\text { Concentration } \\
(\mu g / L)\end{array}$} \\
\hline & & $\mathrm{CHCi}_{3}$ & $\mathrm{BrCHCl}_{2}$ & $\mathrm{CiCHBr}_{2}$ & $\mathrm{CHBr}_{3}$ & $\begin{array}{l}\text { Total } \\
\text { THM }\end{array}$ & NPTOX \\
\hline \multicolumn{8}{|c|}{ REGULAR SAMPLE } \\
\hline 5.50 & 30.0 & 420 & 16.7 & 0.44 & ND & 437 & 1,130 \\
\hline 5.50 & 30.0 & 413 & 16.8 & .43 & ND & 430 & 1,130 \\
\hline 5.50 & 50.0 & 482 & 17.0 & .42 & ND & 499 & 1,170 \\
\hline 5.50 & 50.0 & 486 & 16.9 & .46 & ND & 503 & 1,250 \\
\hline 7.50 & 30.0 & 633 & 22.5 & .67 & ND & 656 & 908 \\
\hline 7.50 & 30.0 & 625 & 21.9 & .63 & ND & 648 & 961 \\
\hline 7.50 & 50.0 & 720 & 21.9 & .63 & ND & 743 & 947 \\
\hline 7.50 & 50.0 & 730 & 22.2 & .64 & ND & 753 & 926 \\
\hline 10.00 & 15.0 & 692 & 21.0 & .78 & ND & 714 & 477 \\
\hline 10.00 & 15.0 & 689 & 20.4 & .76 & ND & 710 & 496 \\
\hline 10.00 & 30.0 & 805 & 23.1 & .74 & ND & 829 & 580 \\
\hline 10.00 & 30.0 & 805 & 23.0 & .76 & ND & 829 & 562 \\
\hline 10.00 & 50.0 & 861 & 23.0 & .73 & ND & 885 & 653 \\
\hline 10.00 & 50.0 & 895 & 23.9 & .76 & ND & 920 & 593 \\
\hline \multicolumn{8}{|c|}{ THAWED SAMPLE } \\
\hline 5.50 & 30.00 & 387 & 16.7 & 0.49 & 0.11 & 404 & 1,080 \\
\hline 5.50 & 30.00 & 390 & 16.4 & .40 & ND & 407 & 1,150 \\
\hline 5.50 & 50.00 & 462 & 16.2 & .40 & ND & 479 & 1,160 \\
\hline 5.50 & 50.00 & 450 & 16.2 & .41 & ND & 467 & 1,160 \\
\hline 7.50 & 30.00 & 596 & 22.0 & .59 & ND & 619 & 832 \\
\hline 7.50 & 30.00 & 591 & 20.3 & .56 & ND & 612 & 825 \\
\hline 7.50 & 50.00 & 696 & 22.7 & .55 & ND & 719 & 914 \\
\hline 7.50 & 50.00 & 694 & 22.1 & .59 & ND & 717 & 883 \\
\hline 10.00 & 30.00 & 820 & 21.8 & .71 & ND & 843 & 571 \\
\hline 10.00 & 30.00 & 818 & 22.6 & .82 & ND & 841 & 562 \\
\hline 10.00 & 50.00 & 860 & 22.0 & .65 & ND & 883 & 603 \\
\hline 10.00 & 50.00 & 888 & 22.6 & .66 & ND & 911 & 656 \\
\hline
\end{tabular}


Table 6.-Concentrations of the four trihalomethane compounds and the total trihalomethane and nonpurgeable total organic-halide concentrations for a reaction time of 168.0 hours as a function of Initial pH and initial free-chlorine concentration, Mississippi River at Quincy, III., April 1, 1992

$[\mu \mathrm{g} / \mathrm{L}$, microgram per liter; $\mathrm{CL}$, free-chlorine concentration; $\mathrm{mg} / \mathrm{L}$, milligram per liter; $\mathrm{CHCl}_{3}$, chloroform; $\mathrm{BrCHCl}_{2}$, bromodichloromethane; $\mathrm{ClCHBr}_{2}$, chlorodibromomethane; $\mathrm{CHBr}_{3}$, bromoform; $\mathrm{THM}$, trihalomethane; NPTOX, nonpurgeable total organic halide; ND, not detected]

\begin{tabular}{|c|c|c|c|c|c|c|c|}
\hline \multirow{2}{*}{ Initial pH } & \multirow{2}{*}{$\begin{array}{c}\text { InitialCL } \\
\text { (mg/L) }\end{array}$} & \multicolumn{6}{|c|}{$\begin{array}{c}\text { Concentration } \\
(\mu g / L)\end{array}$} \\
\hline & & $\mathrm{CHCl}_{3}$ & $\mathrm{BrCHCl}_{2}$ & $\mathrm{ClCHBr}_{2}$ & $\mathrm{CHBr}_{3}$ & $\begin{array}{l}\text { Total } \\
\text { THM }\end{array}$ & NPTOX \\
\hline 5.50 & 15.0 & 248 & 17.4 & 0.82 & ND & 266 & 744 \\
\hline 5.50 & 15.0 & 252 & 17.3 & .72 & ND & 270 & 731 \\
\hline 5.50 & 30.0 & 300 & 16.8 & .64 & ND & 317 & 860 \\
\hline 5.50 & 30.0 & 300 & 17.2 & .69 & ND & 318 & 807 \\
\hline 5.50 & 50.0 & 363 & 17.3 & .78 & ND & 381 & 977 \\
\hline 5.50 & 50.0 & 368 & 17.5 & .71 & ND & 386 & 911 \\
\hline 7.50 & 15.0 & 375 & 22.4 & 1.17 & ND & 399 & 654 \\
\hline 7.50 & 15.0 & 393 & 23.5 & 1.25 & ND & 418 & 688 \\
\hline 7.50 & 30.0 & 392 & 22.4 & 1.17 & ND & 416 & 694 \\
\hline 7.50 & 30.0 & 395 & 21.8 & 1.08 & ND & 418 & 714 \\
\hline 7.50 & 50.0 & 517 & 23.4 & 1.12 & ND & 542 & 838 \\
\hline 7.50 & 50.0 & 514 & 22.8 & 1.10 & ND & 538 & 819 \\
\hline 10.00 & 15.0 & 499 & 23.1 & 1.56 & .03 & 524 & 455 \\
\hline 10.00 & 15.0 & 502 & 23.6 & 1.61 & ND & 527 & 406 \\
\hline 10.00 & 30.0 & 568 & 25.0 & 1.60 & ND & 595 & 401 \\
\hline 10.00 & 30.0 & 561 & 24.9 & 1.54 & ND & 587 & 401 \\
\hline 10.00 & 50.0 & 588 & 24.7 & 1.51 & ND & 614 & 483 \\
\hline 10.00 & 50.0 & 589 & 23.9 & 1.56 & ND & 614 & 525 \\
\hline
\end{tabular}


Table 7.-Concentrations of the four trihalomethane compounds and the total trihalomethane and nonpurgeable total organic-halide concentrations for a reaction time of 168.0 hours as a function of initial $\mathrm{pH}$ and initial free-chlorine concentration, Missouri River 1.6 kilometers upstream from confluence with Mississippi River, March 31, 1992

$[\mu \mathrm{g} / \mathrm{L}$, microgram per liter; $\mathrm{CL}$, free-chlorine concentration; $\mathrm{mg} / \mathrm{L}$, milligram per liter; $\mathrm{CHCl}_{3}$, chloroform; $\mathrm{BrCHCl}_{2}$, bromodichloromethane; $\mathrm{ClCHBr}_{2}$, chlorodibromomethane; $\mathrm{CHBr}_{3}$, bromoform; THM, trihalomethane; NPTOX, nonpurgeable total organic halide]

\begin{tabular}{|c|c|c|c|c|c|c|c|}
\hline \multirow{2}{*}{ Initial pH } & \multirow{2}{*}{$\begin{array}{l}\text { InitialCL } \\
\text { (mg/L) }\end{array}$} & \multicolumn{6}{|c|}{$\begin{array}{c}\text { Concentration } \\
(\mu g / L)\end{array}$} \\
\hline & & $\mathrm{CHCl}_{3}$ & $\mathrm{BrCHCl}_{2}$ & $\mathrm{ClCHBr}_{2}$ & $\mathrm{CHBr}_{3}$ & $\begin{array}{l}\text { Total } \\
\text { THM }\end{array}$ & NPTOX \\
\hline 5.50 & 15.0 & 198 & 41.7 & 7.19 & 0.24 & 247 & 647 \\
\hline 5.50 & 15.0 & 190 & 40.4 & 6.93 & .24 & 238 & 622 \\
\hline 5.50 & 30.0 & 230 & 41.0 & 6.10 & .17 & 277 & 734 \\
\hline 5.50 & 30.0 & 233 & 40.8 & 5.83 & .17 & 280 & 727 \\
\hline 5.50 & 50.0 & 287 & 44.0 & 5.82 & .12 & 337 & 759 \\
\hline 5.50 & 50.0 & 288 & 45.0 & 6.00 & .13 & 339 & 748 \\
\hline 7.50 & 15.0 & 299 & 58.5 & 10.7 & .36 & 369 & 555 \\
\hline 7.50 & 15.0 & 309 & 60.5 & 11.0 & .37 & 381 & 530 \\
\hline 7.50 & 30.0 & 328 & 59.1 & 10.3 & .29 & 398 & 632 \\
\hline 7.50 & 30.0 & 330 & 58.4 & 10.1 & .29 & 399 & 634 \\
\hline 7.50 & 50.0 & 414 & 61.8 & 10.4 & .30 & 486 & 610 \\
\hline 7.50 & 50.0 & 417 & 62.0 & 10.4 & .30 & 490 & 649 \\
\hline 10.00 & 15.0 & 416 & 61.0 & 12.4 & .71 & 490 & 323 \\
\hline 10.00 & 15.0 & 403 & 59.5 & 12.3 & .71 & 476 & .324 \\
\hline 10.00 & 30.0 & 453 & 62.2 & 12.2 & .64 & 528 & 326 \\
\hline 10.00 & 30.0 & 446 & 60.8 & 11.9 & .61 & 519 & 355 \\
\hline 10.00 & 50.0 & 472 & 63.3 & 12.0 & .58 & 548 & 378 \\
\hline 10.00 & 50.0 & 474 & 64.1 & 12.2 & .59 & 551 & 385 \\
\hline
\end{tabular}


Table 8.-Concentrations of the four trihalomethane compounds and the total trlhalomethane and nonpurgeable total organic-hallde concentrations for a reaction time of 168.0 hours as a function of initial pH and initial free-chlorine concentration, Mississippi River at St. Louis, Mo., March 31, 1992

[ $\mu \mathrm{g} / \mathrm{L}$, microgram per liter; $\mathrm{CL}$, free-chlorine concentration; $\mathrm{mg} / \mathrm{L}$, milligram per liter; $\mathrm{CHCl}_{3}$, chloroform; $\mathrm{BrCHCl}_{2}$, bromodichloromethane; $\mathrm{ClCHBr}$, chlorodibromomethane; $\mathrm{CHBr}_{3}$, bromoform; THM, trihalomethane; NPTOX, nonpurgeable total organic halide]

\begin{tabular}{|c|c|c|c|c|c|c|c|}
\hline \multirow{2}{*}{ initial pH } & \multirow{2}{*}{$\begin{array}{c}\text { Initial CL } \\
\text { (mg/L) }\end{array}$} & \multicolumn{6}{|c|}{$\begin{array}{c}\text { Concentration } \\
(\mu g / L)\end{array}$} \\
\hline & & $\mathrm{CHCl}_{3}$ & $\mathrm{BrCHCl}_{2}$ & $\mathrm{ClCHBr}_{2}$ & $\mathrm{CHBr}_{3}$ & $\begin{array}{l}\text { Total } \\
\text { THM }\end{array}$ & NPTOX \\
\hline 5.50 & 15.0 & 221 & 27.7 & 2.81 & 0.10 & 252 & 725 \\
\hline 5.50 & 15.0 & 217 & 28.1 & 2.94 & ND & 248 & 737 \\
\hline 5.50 & 30.0 & 272 & 29.0 & 2.56 & ND & 304 & 858 \\
\hline 5.50 & 30.0 & 276 & 30.0 & 2.82 & ND & 309 & 845 \\
\hline 5.50 & 50.0 & 361 & 32.8 & 2.76 & .01 & 397 & 904 \\
\hline 5.50 & 50.0 & 327 & 31.0 & 2.67 & .01 & 361 & 863 \\
\hline 7.50 & 15.0 & 371 & 41.9 & 4.49 & .07 & 417 & 634 \\
\hline 7.50 & 15.0 & 369 & 41.5 & 4.47 & .07 & 415 & 667 \\
\hline 7.50 & 30.0 & 361 & 41.1 & 4.31 & .06 & 406 & 663 \\
\hline 7.50 & 30.0 & 357 & 39.4 & 4.17 & .06 & 401 & 687 \\
\hline 7.50 & 50.0 & 451 & 42.3 & 4.15 & .06 & 498 & 699 \\
\hline 7.50 & 50.0 & 460 & 43.7 & 4.41 & .09 & 508 & 700 \\
\hline 10.00 & 15.0 & 472 & 42.8 & 5.52 & .17 & 520 & 371 \\
\hline 10.00 & 15.0 & 468 & 42.5 & 5.51 & .17 & 516 & 386 \\
\hline 10.00 & 30.0 & 516 & 43.3 & 5.12 & .14 & 565 & 428 \\
\hline 10.00 & 30.0 & 517 & 44.2 & 5.13 & .14 & 566 & 419 \\
\hline 10.00 & 50.0 & 545 & 44.8 & 5.18 & .13 & 595 & 443 \\
\hline 10.00 & 50.0 & 544 & 44.8 & 5.09 & .14 & 594 & 450 \\
\hline
\end{tabular}


Table 9.-Concentrations of the four trihaiomethane compounds and the total trihalomethane and nonpurgeable total organic-hailde concentrations for a reaction time of $\mathbf{1 6 8 . 0}$ hours as a function of initial pH and initial free-chiorine concentration, Mississippi River at Cairo, III., March 30, 1992

$[\mu \mathrm{g} / \mathrm{L}$, microgram per liter; $\mathrm{CL}$, free-chlorine concentration; $\mathrm{mg} / \mathrm{L}$, milligram per liter; $\mathrm{CHCl}_{3}$, chloroform; $\mathrm{BrCHCl}_{2}$, bromodichloromethane; $\mathrm{ClCHBr}_{2}$, chlorodibromomethane; $\mathrm{CHBr}_{3}$, bromoform; $\mathrm{THM}$, trihalomethane; NPTOX, nonpurgeable total organic halide; $\mathrm{ND}$, not detected]

\begin{tabular}{|c|c|c|c|c|c|c|c|}
\hline \multirow{2}{*}{ Initial pH } & \multirow{2}{*}{$\begin{array}{c}\text { initiai CL } \\
(\mathbf{m g} / \mathrm{L})\end{array}$} & \multicolumn{6}{|c|}{$\begin{array}{c}\text { Concentration } \\
(\mu g / L)\end{array}$} \\
\hline & & $\mathrm{CHCl}_{3}$ & $\mathrm{BrCHCi}_{2}$ & $\mathrm{ClCHBr}_{2}$ & $\mathrm{CHBr}_{3}$ & $\begin{array}{l}\text { Total } \\
\text { THM }\end{array}$ & NPTOX \\
\hline 5.50 & 15.0 & 266 & 23.9 & 1.68 & ND & 292 & 750 \\
\hline 5.50 & 15.0 & 263 & 23.7 & 1.65 & ND & 288 & 760 \\
\hline 5.50 & 30.0 & 298 & 23.3 & 1.41 & ND & 323 & 913 \\
\hline 5.50 & 30.0 & 303 & 23.8 & 1.49 & ND & 328 & 873 \\
\hline 5.50 & 50.0 & 358 & 24.2 & 1.41 & ND & 384 & 914 \\
\hline 5.50 & 50.0 & 360 & 24.3 & 1.43 & ND & 386 & 916 \\
\hline 7.50 & 15.0 & 372 & 32.2 & 2.33 & ND & 407 & 605 \\
\hline 7.50 & 15.0 & 364 & 31.6 & 2.28 & ND & 399 & 597 \\
\hline 7.50 & 30.0 & 410 & 32.4 & 2.22 & ND & 445 & 719 \\
\hline 7.50 & 30.0 & 408 & 32.4 & 2.24 & ND & 443 & 715 \\
\hline 7.50 & 50.0 & 505 & 33.5 & 2.27 & ND & 541 & 700 \\
\hline 7.50 & 50.0 & 505 & 32.9 & 2.22 & ND & 540 & 691 \\
\hline 10.00 & 15.0 & 589 & 36.9 & 2.86 & ND & 629 & 460 \\
\hline 10.00 & 15.0 & 595 & 37.5 & 2.94 & .06 & 636 & 447 \\
\hline 10.00 & 30.0 & 615 & 37.1 & 2.81 & ND & 655 & 441 \\
\hline 10.00 & 30.0 & 616 & 37.1 & 2.78 & ND & 656 & 458 \\
\hline 10.00 & 50.0 & 663 & 37.3 & 2.75 & ND & 703 & 464 \\
\hline 10.00 & 50.0 & 655 & 38.4 & 2.86 & ND & 696 & 496 \\
\hline
\end{tabular}


Table 10.-Concentrations of the four trihalomethane compounds and the total trihalomethane and nonpurgeable total organic-halide concentrations for a reaction time of $\mathbf{1 6 8 . 0}$ hours as a function of initial $\mathrm{pH}$ and initial free-chlorine concentration, Ohio River 1.6 kilometers upstream from confluence with Mississippi River, March 30, 1992

$[\mu \mathrm{g} / \mathrm{L}$, microgram per liter; $\mathrm{CL}$, free-chlorine concentration; $\mathrm{mg} / \mathrm{L}$, milligram per liter; $\mathrm{CHCl}_{3}$, chloroform; $\mathrm{BrCHCl}_{2}$, bromodichloromethane; $\mathrm{ClCHBr}_{2}$, chlorodibromomethane; $\mathrm{CHBr}_{3}$, bromoform; THM, trihalomethane; NPTOX, nonpurgeable total organic halide]

\begin{tabular}{|c|c|c|c|c|c|c|c|}
\hline \multirow{2}{*}{ Initial pH } & \multirow{2}{*}{$\begin{array}{c}\text { Initial CL } \\
\text { (mg/L) }\end{array}$} & \multicolumn{6}{|c|}{$\begin{array}{c}\text { Concentration } \\
(\mu g / L)\end{array}$} \\
\hline & & $\mathrm{CHCl}_{3}$ & $\mathrm{BrCHCl}_{2}$ & $\mathrm{ClCHBr}_{2}$ & $\mathrm{CHBr}_{3}$ & $\begin{array}{l}\text { Total } \\
\text { THM }\end{array}$ & NPTOX \\
\hline 5.50 & 15.0 & 138 & 16.2 & 1.44 & 0.03 & 156 & 392 \\
\hline 5.50 & 15.0 & 143 & 16.9 & 1.50 & .03 & 161 & 391 \\
\hline 5.50 & 30.0 & 171 & 16.4 & 1.36 & .01 & 189 & 451 \\
\hline 5.50 & 30.0 & 172 & 16.0 & 1.32 & .02 & 189 & 448 \\
\hline 5.50 & 50.0 & 229 & 18.2 & 1.49 & .01 & 249 & 477 \\
\hline 5.50 & 50.0 & 226 & 18.1 & 1.44 & .02 & 246 & 475 \\
\hline 7.50 & 15.0 & 205 & 22.0 & 2.29 & .04 & 229 & 323 \\
\hline 7.50 & 15.0 & 208 & 21.9 & 2.25 & .03 & 232 & 341 \\
\hline 7.50 & 30.0 & 234 & 23.3 & 2.29 & .03 & 260 & 343 \\
\hline 7.50 & 30.0 & 241 & 24.2 & 2.35 & .03 & 268 & 357 \\
\hline 7.50 & 50.0 & 273 & 22.3 & 2.21 & .04 & 298 & 360 \\
\hline 7.50 & 50.0 & 277 & 23.2 & 2.27 & .03 & 302 & 355 \\
\hline 10.00 & 15.0 & 304 & 23.6 & 2.71 & .07 & 330 & 216 \\
\hline 10.00 & 15.0 & 303 & 23.0 & 2.63 & .07 & 329 & 217 \\
\hline 10.00 & 30.0 & 328 & 24.1 & 2.61 & .06 & 355 & 233 \\
\hline 10.00 & 30.0 & 323 & 24.7 & 2.63 & .06 & 350 & 242 \\
\hline 10.00 & 50.0 & 360 & 24.4 & 2.48 & .05 & 387 & 311 \\
\hline 10.00 & 50.0 & 372 & 24.9 & 2.52 & .05 & 399 & 275 \\
\hline
\end{tabular}


Table 11.--Concentrations of the four trihalomethane compounds and the total trihalomethane and nonpurgeable total organic-halide concentrations for a reaction time of 168.0 hours as a function of initial pH and initiai free-chlorine concentration, Mississippi River at Memphis, Tenn., March 29, 1992

$[\mu \mathrm{g} / \mathrm{L}$, microgram per liter; $\mathrm{CL}$, free-chlorine concentration; $\mathrm{mg} / \mathrm{L}$, milligram per liter; $\mathrm{CHCl}_{3}$, chloroform; $\mathrm{BrCHCl}_{2}$, bromodichloromethane; $\mathrm{ClCHBr}_{2}$, chlorodibromomethane; $\mathrm{CHBr}_{3}$, bromoform; $\mathrm{THM}$, trihalomethane; NPTOX, nonpurgeable total organic halide]

\begin{tabular}{|c|c|c|c|c|c|c|c|}
\hline \multirow{2}{*}{ Initial pH } & \multirow{2}{*}{$\begin{array}{c}\text { InitialCL } \\
\text { (mg/l) }\end{array}$} & \multicolumn{6}{|c|}{$\begin{array}{c}\text { Concentration } \\
(\mu g / L)\end{array}$} \\
\hline & & $\mathrm{CHCl}_{3}$ & $\mathrm{BrCHCl}_{2}$ & $\mathrm{ClCHBr}_{2}$ & $\mathrm{CHBr}_{3}$ & $\begin{array}{l}\text { Total } \\
\text { THM }\end{array}$ & NPTOX \\
\hline 5.50 & 15.0 & 191 & 17.4 & 1.37 & $\overline{\mathrm{ND}}$ & 210 & 575 \\
\hline 5.50 & 15.0 & 206 & 18.2 & 1.41 & ND & 226 & 573 \\
\hline 5.50 & 30.0 & 228 & 17.5 & 1.19 & ND & 247 & 611 \\
\hline 5.50 & 30.0 & 220 & 16.9 & 1.18 & ND & 238 & 621 \\
\hline 5.50 & 50.0 & 288 & 19.5 & 1.30 & .01 & 309 & 658 \\
\hline 5.50 & 50.0 & 285 & 18.8 & 1.25 & ND & 305 & 719 \\
\hline 7.50 & 15.0 & ${ }^{1} 289$ & ${ }^{1} 24.8$ & $1_{2.06}$ & ${ }^{1} .02$ & $1_{316}$ & $1_{473}$ \\
\hline 7.50 & 15.0 & $1_{284}$ & $1_{24.0}$ & $1_{2.00}$ & ${ }^{1} .02$ & $1_{310}$ & ${ }^{1} 480$ \\
\hline 7.50 & 30.0 & 314 & 25.9 & 2.05 & .02 & 342 & 498 \\
\hline 7.50 & 30.0 & 325 & 26.4 & 2.07 & .02 & 353 & 528 \\
\hline 7.50 & 50.0 & $2_{393}$ & ${ }^{2} 25.9$ & ${ }^{2} 2.04$ & ${ }^{2} .02$ & ${ }^{2} 421$ & 2496 \\
\hline 7.50 & 50.0 & 2385 & ${ }^{2} 25.3$ & ${ }^{2} 1.97$ & ${ }^{2} .02$ & 2412 & 2521 \\
\hline 10.00 & 15.0 & 424 & 27.5 & 2.41 & .04 & 454 & 303 \\
\hline 10.00 & 15.0 & 426 & 27.3 & 2.37 & .04 & 456 & 288 \\
\hline 10.00 & 30.0 & 470 & 28.1 & 2.35 & .04 & 500 & 316 \\
\hline 10.00 & 30.0 & 466 & 28.0 & 2.34 & .04 & 496 & 319 \\
\hline 10.00 & 50.0 & 480 & 27.3 & 2.16 & .03 & 509 & 339 \\
\hline 10.00 & 50.0 & 500 & 28.6 & 2.27 & .04 & 531 & 346 \\
\hline
\end{tabular}

${ }^{1}$ Reaction time of 168.3 hours rather than 168.0 hours.

${ }^{2}$ Reaction time of 168.2 hours rather than 168.0 hours. 
Tabie 12.-Concentrations of the four trihaiomethane compounds and the total trihaiomethane and nonpurgeabie totai organic-haiide concentrations for a reaction time of 168.0 hours as a function of initial pH and initial free-chiorine concentration, Mississippi River at Greenvilie, Miss., March 28, 1992

$[\mu \mathrm{g} / \mathrm{L}$, microgram per liter; $\mathrm{CL}$, free-chlorine concentration; $\mathrm{mg} / \mathrm{L}$, milligram per liter; $\mathrm{CHCl}_{3}$, chloroform; $\mathrm{BrCHCl}_{2}$, bromodichloromethane; $\mathrm{ClCHBr}_{2}$, chlorodibromomethane; $\mathrm{CHBr}_{3}$, bromoform; THM, trihalomethane; NPTOX, nonpurgeable total organic halide]

\begin{tabular}{|c|c|c|c|c|c|c|c|}
\hline \multirow{2}{*}{ initiai pH } & \multirow{2}{*}{$\begin{array}{c}\text { initial CL } \\
\text { (mg/L) }\end{array}$} & \multicolumn{6}{|c|}{$\begin{array}{c}\text { Concentration } \\
(\mu g / L)\end{array}$} \\
\hline & & $\mathrm{CHCl}_{3}$ & $\mathrm{BrCHCl}_{2}$ & $\mathrm{ClCHBr}_{2}$ & $\mathrm{CHBr}_{3}$ & $\begin{array}{l}\text { Total } \\
\text { THM }\end{array}$ & NPTOX \\
\hline 5.50 & 15.0 & 170 & 18.6 & 1.39 & $\overline{\mathrm{ND}}$ & 190 & 438 \\
\hline 5.50 & 15.0 & 173 & 19.2 & 1.47 & ND & 194 & 459 \\
\hline 5.50 & 30.0 & 220 & 19.8 & 1.37 & .01 & 241 & 563 \\
\hline 5.50 & 30.0 & 217 & 19.2 & 1.35 & .02 & 238 & 577 \\
\hline 5.50 & 50.0 & 262 & 20.2 & 1.37 & .01 & 284 & 616 \\
\hline 5.50 & 50.0 & 260 & 20.4 & 1.36 & .01 & 282 & 618 \\
\hline 7.50 & 15.0 & 268 & 26.1 & 2.37 & .03 & 296 & 461 \\
\hline 7.50 & 15.0 & 273 & 26.2 & 2.38 & .03 & 302 & 452 \\
\hline 7.50 & 30.0 & 308 & 27.9 & 2.40 & .03 & 338 & 483 \\
\hline 7.50 & 30.0 & 304 & 26.8 & 2.28 & .03 & 333 & 474 \\
\hline 7.50 & 50.0 & 372 & 27.0 & 2.23 & .03 & 401 & 485 \\
\hline 7.50 & 50.0 & 379 & 27.5 & 2.27 & .03 & 409 & 545 \\
\hline 10.00 & 15.0 & 398 & 29.2 & 2.96 & .07 & 430 & 288 \\
\hline 10.00 & 15.0 & 392 & 27.7 & 2.75 & .06 & 423 & 294 \\
\hline 10.00 & 30.0 & 444 & 29.4 & 2.74 & .06 & 476 & 271 \\
\hline 10.00 & 30.0 & 454 & 30.1 & 2.78 & .05 & 487 & 298 \\
\hline 10.00 & 50.0 & 478 & 30.7 & 2.79 & .05 & 512 & 294 \\
\hline 10.00 & 50.0 & 499 & 31.0 & 2.79 & .05 & 533 & 307 \\
\hline
\end{tabular}


Tabie 13.--Concentrations of the four trihaiomethane compounds and the total trihaiomethane and nonpurgeable totai organic-halide concentrations for a reaction time of $\mathbf{1 6 8 . 0}$ hours as a function of initial pH and initial free-chlorine concentration, Mississippi River at Natchez, Miss., March 26, 1992

$[\mu \mathrm{g} / \mathrm{L}$, microgram per liter; $\mathrm{CL}$, free-chlorine concentration; $\mathrm{mg} / \mathrm{L}$, milligram per liter; $\mathrm{CHCl}_{3}$, chloroform; $\mathrm{BrCHCl}_{2}$, bromodichloromethane; $\mathrm{ClCHBr}_{2}$, chlorodibromomethane; $\mathrm{CHBr}_{3}$, bromoform; THM, trihalomethane; NPTOX, nonpurgeable total organic halide]

\begin{tabular}{|c|c|c|c|c|c|c|c|}
\hline \multirow{2}{*}{ Initial pH } & \multirow{2}{*}{$\begin{array}{c}\text { Initial CL } \\
\text { (mg/L) }\end{array}$} & \multicolumn{6}{|c|}{$\begin{array}{c}\text { Concentration } \\
(\mu g / L)\end{array}$} \\
\hline & & $\mathrm{CHCl}_{3}$ & $\mathrm{BrCHCl}_{2}$ & $\mathrm{ClCHBr}_{2}$ & $\mathrm{CHBr}_{3}$ & $\begin{array}{l}\text { Total } \\
\text { THM }\end{array}$ & NPTOX \\
\hline 5.50 & 15.0 & 186 & 19.6 & 1.62 & $\mathrm{ND}$ & 207 & 512 \\
\hline 5.50 & 15.0 & 183 & 18.7 & 1.52 & ND & 203 & 529 \\
\hline 5.50 & 30.0 & 220 & 19.2 & 1.60 & ND & 241 & 564 \\
\hline 5.50 & 30.0 & 224 & 20.2 & 1.60 & .02 & 246 & 571 \\
\hline 5.50 & 50.0 & 271 & 20.3 & 1.48 & .02 & 293 & 605 \\
\hline 5.50 & 50.0 & 271 & 20.1 & 1.51 & .01 & 293 & 612 \\
\hline 7.50 & 15.0 & 274 & 26.6 & 2.42 & .03 & 303 & 421 \\
\hline 7.50 & 15.0 & 271 & 26.2 & 2.39 & .03 & 300 & 434 \\
\hline 7.50 & 30.0 & 313 & 26.7 & 2.34 & .02 & 342 & 472 \\
\hline 7.50 & 30.0 & 318 & 27.1 & 2.33 & ND & 347 & 481 \\
\hline 7.50 & 50.0 & 369 & 26.9 & 2.29 & .02 & 398 & 471 \\
\hline 7.50 & 50.0 & 362 & 26.4 & 2.24 & .02 & 391 & 463 \\
\hline 10.00 & 15.0 & 399 & 28.8 & 2.99 & .07 & 431 & 299 \\
\hline 10.00 & 15.0 & 397 & 28.6 & 2.96 & .08 & 429 & 286 \\
\hline 10.00 & 30.0 & 427 & 28.5 & 2.75 & .06 & 458 & 315 \\
\hline 10.00 & 30.0 & 434 & 30.6 & 3.23 & .07 & 468 & 312 \\
\hline 10.00 & 50.0 & 468 & 30.0 & 2.83 & .06 & 501 & 333 \\
\hline 10.00 & 50.0 & 476 & 29.9 & 2.80 & .06 & 509 & 354 \\
\hline
\end{tabular}


Tabie 14.-Concentrations of the four trihaiomethane compounds and the total trihalomethane and nonpurgeable total organlc-halide concentrations for a reaction time of 168.0 hours as a function of initial $\mathrm{pH}$ and initial free-chlorine concentration, Mississippi River at Baton Rouge, La., March 26, 1992

$[\mu \mathrm{g} / \mathrm{L}$, microgram per liter; $\mathrm{CL}$, free-chlorine concentration; $\mathrm{mg} / \mathrm{L}$, milligram per liter; $\mathrm{CHCl}_{3}$, chloroform; $\mathrm{BrCHCl}_{2}$, bromodichloromethane; $\mathrm{ClCHBr}_{2}$, chlorodibromomethane; $\mathrm{CHBr}_{3}$, bromoform; $\mathrm{THM}$, trihalomethane; NPTOX, nonpurgeable total organic halide]

\begin{tabular}{|c|c|c|c|c|c|c|c|}
\hline \multirow{2}{*}{ Initial pH } & \multirow{2}{*}{$\begin{array}{l}\text { Initiai CL } \\
\text { (mg/L) }\end{array}$} & \multicolumn{6}{|c|}{$\begin{array}{c}\text { Concentration } \\
(\mu \mathrm{g} / \mathrm{L})\end{array}$} \\
\hline & & $\mathrm{CHCl}_{3}$ & $\mathrm{BrCHCl}_{2}$ & $\mathrm{CiCHBr}_{2}$ & $\mathrm{CHBr}_{3}$ & $\begin{array}{l}\text { Total } \\
\text { THM }\end{array}$ & NPTOX \\
\hline 5.50 & 15.0 & 189 & 19.3 & 1.62 & $\overline{N D}$ & 210 & 516 \\
\hline 5.50 & 15.0 & 186 & 19.0 & 1.62 & ND & 207 & 531 \\
\hline 5.50 & 30.0 & 213 & 18.6 & 1.43 & ND & 233 & 569 \\
\hline 5.50 & 30.0 & 216 & 18.8 & 1.46 & ND & 236 & 580 \\
\hline 5.50 & 50.0 & 268 & 19.6 & 1.55 & .01 & 289 & 582 \\
\hline 5.50 & 50.0 & 266 & 19.1 & 1.45 & .01 & 287 & 620 \\
\hline 7.50 & 15.0 & 274 & 25.6 & 2.43 & ND & 302 & 428 \\
\hline 7.50 & 15.0 & 278 & 26.2 & 2.48 & .04 & 307 & 427 \\
\hline 7.50 & 30.0 & 345 & 25.8 & 2.34 & .01 & 373 & 472 \\
\hline 7.50 & 30.0 & 323 & 25.9 & 2.34 & ND & 351 & 449 \\
\hline 7.50 & 50.0 & 362 & 25.3 & 2.28 & .03 & 390 & 455 \\
\hline 7.50 & 50.0 & 362 & 25.6 & 2.29 & .03 & 390 & 474 \\
\hline 10.00 & 15.0 & 396 & 27.1 & 2.84 & .07 & 426 & 278 \\
\hline 10.00 & 15.0 & 402 & 27.4 & 2.92 & .07 & 432 & 286 \\
\hline 10.00 & 30.0 & 431 & 28.0 & 2.79 & .06 & 462 & 290 \\
\hline 10.00 & 30.0 & 437 & 28.1 & 2.80 & .06 & 468 & 313 \\
\hline 10.00 & 50.0 & 459 & 27.8 & 2.68 & .06 & 490 & 329 \\
\hline 10.00 & 50.0 & 468 & 28.4 & 2.74 & ND & 499 & 336 \\
\hline
\end{tabular}


Table 15.-Concentrations of the four trihalomethane compounds and the total trihalomethane and nonpurgeable total organic-halide concentrations for a reaction time of $\mathbf{1 6 8 . 0}$ hours as a function of initial pH and initial free-chlorine concentration, Mississippi River at New Orleans, La., March 25, 1992

$[\mu \mathrm{g} / \mathrm{L}$, microgram per liter; $\mathrm{CL}$, free-chlorine concentration; $\mathrm{mg} / \mathrm{L}$, milligram per liter; $\mathrm{CHCl}_{3}$, chloroform; $\mathrm{BrCHCl}_{2}$, bromodichloromethane; $\mathrm{ClCHBr}_{2}$, chlorodibromomethane; $\mathrm{CHBr}_{3}$, bromoform; $\mathrm{THM}$, trihalomethane; NPTOX, nonpurgeable total organic halide]

\begin{tabular}{|c|c|c|c|c|c|c|c|}
\hline \multirow{2}{*}{ Initial pH } & \multirow{2}{*}{$\begin{array}{c}\text { initial CL } \\
\text { (mg/L) }\end{array}$} & \multicolumn{6}{|c|}{$\begin{array}{c}\text { Concentration } \\
(\mu g / L)\end{array}$} \\
\hline & & $\mathrm{CHCl}_{3}$ & $\mathrm{BrCHCl}_{2}$ & $\mathrm{ClCHBr}_{2}$ & $\mathrm{CHBr}_{3}$ & $\begin{array}{l}\text { Total } \\
\text { THM }\end{array}$ & NPTOX \\
\hline 5.50 & 15.0 & 161 & 31.0 & 5.35 & 0.13 & 197 & 466 \\
\hline 5.50 & 15.0 & 160 & 30.3 & 4.93 & .12 & 195 & 489 \\
\hline 5.50 & 30.0 & 183 & 29.9 & 4.34 & .09 & 217 & 515 \\
\hline 5.50 & 30.0 & 180 & 29.0 & 4.10 & .09 & 213 & 514 \\
\hline 5.50 & 50.0 & 220 & 29.8 & 3.89 & .07 & 254 & 528 \\
\hline 5.50 & 50.0 & 226 & 31.3 & 4.10 & .08 & 261 & 542 \\
\hline 7.50 & 15.0 & 225 & 41.4 & 7.72 & .23 & 274 & 402 \\
\hline 7.50 & 15.0 & 227 & 42.3 & 7.85 & .25 & 277 & 363 \\
\hline 7.50 & 30.0 & 245 & 40.3 & 7.45 & .19 & 293 & 401 \\
\hline 7.50 & 30.0 & 254 & 40.9 & 7.54 & .19 & 303 & 401 \\
\hline 7.50 & 50.0 & 307 & 44.4 & 7.51 & .21 & 359 & 387 \\
\hline 7.50 & 50.0 & 306 & 43.8 & 7.47 & .21 & 357 & 399 \\
\hline 10.00 & 15.0 & 322 & 42.6 & 8.68 & .44 & 374 & 252 \\
\hline 10.00 & 15.0 & 327 & 44.5 & 8.93 & .45 & 381 & 256 \\
\hline 10.00 & 30.0 & 359 & 45.2 & 8.65 & .40 & 413 & 278 \\
\hline 10.00 & 30.0 & 360 & 45.3 & 8.70 & .40 & 414 & 272 \\
\hline 10.00 & 50.0 & 379 & 44.9 & 8.17 & .35 & 432 & 300 \\
\hline 10.00 & 50.0 & 386 & 47.0 & 8.56 & .36 & 442 & 295 \\
\hline
\end{tabular}


Table 16.-Concentrations of the four trihalomethane compounds and the total trihalomethane and nonpurgeable total organic-halide concentrations as a function of reaction time for an initial pH of 7.50 and an initial free-chlorine concentration of 30.0 milligrams per liter, Mississippi River at LaCrosse, Wis., April 3, 1992

[ $\mu \mathrm{g} / \mathrm{L}$, microgram per liter; $\mathrm{CHCl}_{3}$, chloroform; $\mathrm{BrCHCl}_{2}$, bromodichloromethane;

$\mathrm{ClCHBr}$, chlorodibromomethane; $\mathrm{CHBr}_{3}$, bromoform; $\mathrm{THM}$, trihalomethane; NPTOX, nonpurgeable total organic halide; ND, not detected]

\begin{tabular}{ccccccc}
\hline $\begin{array}{c}\text { Reaction } \\
\text { time } \\
\text { (hours) }\end{array}$ & $\mathrm{CHCl}_{3}$ & $\mathrm{BrCHCl}_{2}$ & $\mathrm{CICHBr}_{2}$ & $\mathrm{CHBr}_{3}$ & Total THM & NPTOX \\
\cline { 2 - 7 } & 34.6 & 1.91 & 0.07 & $\mathrm{ND}$ & 36.6 & 158 \\
0.00 & 147 & 10.7 & .35 & $\mathrm{ND}$ & 158 & 494 \\
2.00 & 190 & 12.8 & .42 & ND & 203 & 706 \\
6.00 & 234 & 14.8 & .43 & ND & 249 & 745 \\
14.00 & 292 & 17.5 & .55 & ND & 310 & 877 \\
24.00 & & & & & & \\
& & & .63 & ND & 372 & 979 \\
48.00 & 352 & 18.9 & .70 & ND & 437 & 974 \\
72.00 & 415 & 21.3 & .71 & ND & 509 & 1,110 \\
126.0 & 486 & 22.0 & .71 & ND & 560 & 1,100 \\
168.0 & 536 & 22.8 & .79 & ND & 572 & 1,070 \\
168.0 & 547 & 24.0 & .79 &
\end{tabular}

Table 17.-Concentrations of the four trihalomethane compounds and the total trihalomethane and nonpurgeable total organic-halide concentrations as a function of reaction time for an initial pH of 7.50 and an initiai free-chlorine concentration of 30.0 milligrams per liter, Mississippi River at Dubuque, lowa, April 2, 1992

$\left[\mu \mathrm{g} / \mathrm{L}\right.$, microgram per liter; $\mathrm{CHCl}_{3}$, chloroform; $\mathrm{BrCHCl}_{2}$, bromodichloromethane; $\mathrm{ClCHBr}_{2}$, chlorodibromomethane; $\mathrm{CHBr}_{3}$, bromoform; $\mathrm{THM}$, trihalomethane; NPTOX, nonpurgeable total organic halide; ND, not detected]

\begin{tabular}{ccccccc}
\hline $\begin{array}{c}\text { Reaction } \\
\text { time } \\
\text { (hours) }\end{array}$ & $\mathrm{CHCl}_{3}$ & $\mathrm{BrCHCl}_{2}$ & $\mathrm{ClCHBr}_{2}$ & $\mathrm{CHBr}_{3}$ & Total THM & NPTOX \\
\hline 0.00 & 38.1 & 1.94 & 0.03 & $\mathrm{ND}$ & 40.1 & 151 \\
2.00 & 160 & 9.43 & .25 & ND & 170 & 432 \\
6.00 & 202 & 11.5 & .31 & ND & 214 & 615 \\
12.18 & 244 & 13.2 & .39 & ND & 258 & 670 \\
24.00 & 304 & 15.3 & .44 & ND & 320 & 731 \\
& & & & & & \\
48.00 & 392 & 18.0 & .54 & ND & 411 & 820 \\
72.00 & 435 & 18.4 & .54 & ND & 454 & 858 \\
121.0 & 490 & 18.8 & .55 & ND & 509 & 970 \\
168.0 & 553 & 19.9 & .57 & ND & 573 & 1,020 \\
168.0 & 557 & 20.0 & .55 & ND & 578 & 1,050 \\
\hline
\end{tabular}


Table 18.-Concentrations of the four trihalomethane compounds and the total trihaiomethane and nonpurgeabie total organic-halide concentrations as a function of reaction time for an initial pH of 7.50 and an initial free-chlorine concentration of 30.0 miiligrams per iiter, Mississippi River at Quincy, ili., Aprii 1, 1992

[ $\mu \mathrm{g} / \mathrm{L}$, microgram per liter; $\mathrm{CHCl}_{3}$, chloroform; $\mathrm{BrCHCl}_{2}$, bromodichloromethane; $\mathrm{ClCHBr}$, chlorodibromomethane; $\mathrm{CHBr}_{3}$, bromoform; $\mathrm{THM}$, trihalomethane; NPTOX, nonpurgeable total organic halide; ND, not detected]

\begin{tabular}{ccccccc}
\hline $\begin{array}{c}\text { Reaction } \\
\text { time } \\
\text { (hours) }\end{array}$ & $\mathrm{CHCl}_{3}$ & $\mathrm{BrCHCi}_{2}$ & CiCHBr $_{2}$ & $\mathrm{CHBr}_{3}$ & Totai THM & NPTOX \\
\cline { 2 - 7 } & 27.6 & 2.06 & 0.13 & $\mathrm{ND}$ & 29.8 & 98.9 \\
\hline 0.00 & 11.1 & .57 & $\mathrm{ND}$ & 113 & 297 \\
2.00 & 101 & 13.2 & .72 & $\mathrm{ND}$ & 147 & 462 \\
6.00 & 133 & 15.2 & .81 & $\mathrm{ND}$ & 186 & 491 \\
13.00 & 170 & 16.7 & .88 & $\mathrm{ND}$ & 221 & 523 \\
24.00 & 203 & & & & & \\
& & & & ND & 279 & 626 \\
48.00 & 259 & 18.6 & 1.00 & $\mathrm{ND}$ & 325 & 616 \\
72.00 & 304 & 20.2 & 1.08 & $\mathrm{ND}$ & 367 & 650 \\
120.0 & 345 & 21.2 & 1.15 & $\mathrm{ND}$ & 416 & 694 \\
168.0 & 392 & 22.4 & 1.17 & $\mathrm{ND}$ & 418 & 714 \\
168.0 & 395 & 21.8 & 1.08 & & \\
\hline
\end{tabular}

Table 19.-Concentrations of the four trihaiomethane compounds and the total trihaiomethane and nonpurgeable total organic-hailde concentrations as a function of reaction time for an initial $\mathrm{pH}$ of 7.50 and an initiai free-chlorine concentration of 30.0 miliigrams per iiter, Missouri River 1.6 kilometers upstream from confluence with Mississippi River, March 31, 1992

$\left[\mu \mathrm{g} / \mathrm{L}\right.$, microgram per liter; $\mathrm{CHCl}_{3}$, chloroform; $\mathrm{BrCHCl}_{2}$, bromodichloromethane; $\mathrm{ClCHBr}_{2}$, chlorodibromomethane; $\mathrm{CHBr}_{3}$, bromoform; $\mathrm{THM}$, trihalomethane; NPTOX, nonpurgeable total organic halide; ND, not detected]

\begin{tabular}{ccccccc}
\hline $\begin{array}{c}\text { Reactlon } \\
\text { time } \\
\text { (hours) }\end{array}$ & $\mathrm{CHCl}_{3}$ & $\mathrm{BrCHCl}_{2}$ & ClCHBr $_{2}$ & $\mathrm{CHBr}_{3}$ & Total THM & NPTOX \\
\cline { 2 - 7 } & 18.4 & 5.06 & 1.35 & 0.05 & 24.9 & 138 \\
0.00 & 73.5 & 26.1 & 5.96 & .18 & 106 & 286 \\
2.00 & 97.5 & 35.6 & 8.01 & .24 & 141 & 346 \\
6.00 & 125 & 39.0 & 8.30 & .25 & 173 & 421 \\
12.00 & 166 & 44.8 & 9.03 & .27 & 220 & 446 \\
24.00 & & & & & & \\
& 213 & 48.9 & 9.66 & .27 & 272 & 524 \\
49.00 & 248 & 53.3 & 10.0 & .27 & 312 & 566 \\
71.00 & 278 & 51.7 & 9.25 & .25 & 339 & 552 \\
121.0 & 328 & 59.1 & 10.3 & .29 & 398 & 632 \\
168.0 & 330 & 58.4 & 10.1 & .29 & 399 & 634 \\
168.0 & & & & & & \\
\hline
\end{tabular}


Table 20.-Concentrations of the four trihalomethane compounds and the total trihalomethane and nonpurgeable total organic-halide concentrations as a function of reaction time for an initial pH of 7.50 and an initial free-chlorine concentration of 30.0 milligrams per liter, Mississippi River at St. Louis, Mo., March 31, 1992

[ $\mu \mathrm{g} / \mathrm{L}$, microgram per liter; $\mathrm{CHCl}_{3}$, chloroform; $\mathrm{BrCHCl}_{2}$, bromodichloromethane; $\mathrm{ClCHBr}_{2}$, chlorodibromomethane; $\mathrm{CHBr}_{3}$, bromoform; $\mathrm{THM}$, trihalomethane; NPTOX, nonpurgeable total organic halide; ND, not detected]

\begin{tabular}{ccccccc}
\hline $\begin{array}{c}\text { Reaction } \\
\text { time } \\
\text { (hours) }\end{array}$ & $\mathrm{CHCl}_{3}$ & $\mathrm{BrCHCi}_{2}$ & ClCHBr $_{2}$ & $\mathrm{CHBr}_{3}$ & Total THM & NPTOX \\
\hline 0.00 & 21.5 & 3.32 & 0.50 & $\mathrm{ND}$ & 25.3 & 85.8 \\
2.00 & 90.1 & 19.9 & 2.47 & .04 & 113 & 216 \\
6.00 & 120 & 24.2 & 3.07 & .05 & 147 & 366 \\
12.00 & 151 & 27.8 & 3.62 & .06 & 182 & 412 \\
24.00 & 189 & 30.7 & 3.67 & $\mathrm{ND}$ & 223 & 462 \\
& & & & & & \\
48.00 & 240 & 35.4 & 4.09 & .06 & 280 & 485 \\
72.00 & 272 & 36.9 & 4.12 & .06 & 313 & 594 \\
121.0 & 329 & 41.1 & 4.63 & .06 & 375 & 631 \\
168.0 & 361 & 41.1 & 4.31 & .06 & 406 & 663 \\
168.0 & 357 & 39.4 & 4.17 & .06 & 401 & 687 \\
\hline
\end{tabular}

Tabie 21.-Concentrations of the four trihalomethane compounds and the total trihalomethane and nonpurgeable total organic-halide concentrations as a function of reaction time for an initial pH of 7.50 and an initiai free-chlorine concentration of 30.0 milligrams per liter, Mississippi River at Cairo, ill., March 30, 1992

[ $\mu \mathrm{g} / \mathrm{L}$, microgram per liter; $\mathrm{CHCl}_{3}$, chloroform; $\mathrm{BrCHCl}_{2}$, bromodichloromethane; $\mathrm{ClCHBr}_{2}$, chlorodibromomethane; $\mathrm{CHBr}_{3}$, bromoform; $\mathrm{THM}$, trihalomethane; NPTOX, nonpurgeable total organic halide]

\begin{tabular}{ccccccc}
\hline $\begin{array}{c}\text { Reaction } \\
\text { time } \\
\text { (hours) }\end{array}$ & $\mathrm{CHCl}_{3}$ & $\mathrm{BrCHCl}_{2}$ & ClCHBr $_{2}$ & $\mathrm{CHBr}_{3}$ & Total THM & NPTOX \\
\cline { 2 - 7 } & 28.2 & 2.91 & 0.29 & ND & 31.4 & 118 \\
\hline 0.00 & 100 & 14.3 & 1.23 & .02 & 116 & 320 \\
2.00 & 139 & 19.3 & 1.60 & .03 & 160 & 411 \\
6.00 & 162 & 20.6 & 1.70 & .02 & 184 & 509 \\
12.00 & 208 & 24.2 & 1.91 & .02 & 234 & 566 \\
24.00 & 208 & & & & & \\
& & 25.8 & 1.89 & .01 & 294 & 589 \\
49.00 & 266 & 28.0 & 2.01 & .02 & 321 & 654 \\
72.00 & 291 & 30.6 & 2.25 & .02 & 381 & 713 \\
126.0 & 348 & 32.4 & 2.22 & ND & 445 & 719 \\
168.0 & 410 & 32.4 & 2.24 & ND & 443 & 715 \\
168.0 & 408 & & & & & \\
\hline
\end{tabular}


Table 22.-Concentrations of the four trihalomethane compounds and the total trihalomethane and nonpurgeable total organic-halide concentrations as a function of reaction time for an initial pH of 7.50 and an initial free-chlorine concentration of 30.0 miligrams per liter, Ohio River 1.6 kilometers upstream from confluence with Mississippi River, March 30, 1992

[ $\mu \mathrm{g} / \mathrm{L}$, microgram per liter; $\mathrm{CHCl}_{3}$, chloroform; $\mathrm{BrCHCl}_{2}$, bromodichloromethane; $\mathrm{ClCHBr}_{2}$, chlorodibromomethane; $\mathrm{CHBr}_{3}$, bromoform; $\mathrm{THM}$, trihalomethane; NPTOX, nonpurgeable total organic halide]

\begin{tabular}{ccccccc}
\hline $\begin{array}{c}\text { Reaction } \\
\text { time } \\
\text { (hours) }\end{array}$ & $\mathrm{CHCi}_{3}$ & $\mathrm{BrCHCl}_{2}$ & $\mathrm{CiCHBr}_{2}$ & $\mathrm{CHBr}_{3}$ & Total THM & NPTOX \\
\cline { 2 - 7 } & 14.0 & 1.78 & 0.17 & $\mathrm{ND}$ & 16.0 & 66.9 \\
\hline 0.00 & 59.3 & 11.2 & 1.30 & .02 & 71.8 & 177 \\
3.00 & 69.8 & 13.1 & 1.53 & .02 & 84.4 & 235 \\
6.00 & 86.4 & 15.1 & 1.76 & .03 & 103 & 227 \\
12.00 & 109 & 17.2 & 1.96 & .02 & 128 & 266 \\
22.50 & & & & & & \\
& 143 & 19.1 & 2.05 & .03 & 164 & 298 \\
48.00 & 172 & 20.3 & 2.13 & .03 & 194 & 313 \\
72.00 & 208 & 21.8 & 2.18 & .03 & 232 & 345 \\
123.00 & 234 & 23.3 & 2.29 & .03 & 260 & 343 \\
168.00 & 241 & 24.2 & 2.35 & .03 & 268 & 357 \\
168.00 & 24.2 & &
\end{tabular}

Table 23.--Concentrations of the four trihalomethane compounds and the totai trihalomethane and nonpurgeable total organic-halide concentrations as a function of reaction time for an initial pH of 7.50 and an initial free-chlorine concentration of 30.0 milligrams per liter, Mississippi River at Memphis, Tenn., March 29, 1992

[ $\mu \mathrm{g} / \mathrm{L}$, microgram per liter; $\mathrm{CHCl}_{3}$, chloroform; $\mathrm{BrCHCl}_{2}$, bromodichloromethane; $\mathrm{ClCHBr}_{2}$, chlorodibromomethane; $\mathrm{CHBr}_{3}$, bromoform; $\mathrm{THM}$, trihalomethane; NPTOX, nonpurgeable total organic halide]

\begin{tabular}{ccccccc}
\hline $\begin{array}{c}\text { Reaction } \\
\text { time } \\
\text { (hours) }\end{array}$ & $\mathrm{CHCl}_{3}$ & $\mathrm{BrCHCl}_{2}$ & CiCHBr $_{2}$ & $\mathrm{CHBr}_{3}$ & Total THM & NPTOX \\
\cline { 2 - 7 } & 23.2 & 2.86 & 0.26 & $\mathrm{ND}$ & 26.3 & 102 \\
\hline 0.00 & 71.8 & 10.6 & .98 & $\mathrm{ND}$ & 83.4 & 238 \\
2.00 & 97.5 & 14.4 & 1.37 & $\mathrm{ND}$ & 113 & 305 \\
6.00 & 16.4 & 16.4 & 1.56 & .01 & 143 & 348 \\
13.00 & 125 & 18.2 & 1.66 & .01 & 174 & 402 \\
24.00 & 154 & & & & & \\
& & 20.5 & 1.74 & .02 & 220 & 460 \\
48.00 & 198 & 21.8 & 1.79 & .02 & 253 & 486 \\
72.00 & 229 & 24.2 & 2.07 & .02 & 308 & 521 \\
126.0 & 282 & 25.9 & 2.05 & .02 & 342 & 498 \\
168.00 & 314 & 26.4 & 2.07 & .02 & 353 & 528 \\
168.0 & 325 & & & & & \\
\hline
\end{tabular}


Table 24.-Concentrations of the four trihaiomethane compounds and the total trihalomethane and nonpurgeable total organic-halide concentrations as a function of reaction time for an initial pH of 7.50 and an initial free-chlorine concentration of 30.0 milligrams per liter, Mississippi River at Greenville, Miss., March 28, 1992

$\left[\mu \mathrm{g} / \mathrm{L}\right.$, microgram per liter; $\mathrm{CHCl}_{3}$, chloroform; $\mathrm{BrCHCl}_{2}$, bromodichloromethane; $\mathrm{ClCHBr}_{2}$, chlorodibromomethane; $\mathrm{CHBr}_{3}$, bromoform; $\mathrm{THM}$, trihalomethane;

NPTOX, nonpurgeable total organic halide]

\begin{tabular}{ccccccc}
\hline $\begin{array}{c}\text { Reaction } \\
\text { time } \\
\text { (hours) }\end{array}$ & $\mathrm{CHCl}_{3}$ & $\mathrm{BrCHCl}_{2}$ & ClCHBr $_{2}$ & $\mathrm{CHBr}_{3}$ & Total THM & NPTOX \\
\cline { 2 - 7 } & 20.3 & 2.57 & 0.23 & $\mathrm{ND}$ & 23.1 & 89.9 \\
\hline 0.00 & 76.5 & 13.2 & 1.31 & .01 & 91.0 & 233 \\
2.00 & 97.4 & 16.3 & 1.67 & .02 & 115 & 307 \\
6.00 & 120 & 18.2 & 1.87 & .02 & 140 & 335 \\
12.00 & 148 & 20.1 & 1.94 & .02 & 170 & 356 \\
24.00 & & & & & & \\
& 192 & 23.0 & 2.14 & .02 & 217 & 380 \\
48.00 & 223 & 24.6 & 2.23 & .03 & 250 & 441 \\
72.00 & 264 & 26.0 & 2.27 & $\mathrm{ND}$ & 292 & 460 \\
121.0 & 308 & 27.9 & 2.40 & .03 & 338 & 483 \\
168.0 & 304 & 26.8 & 2.28 & .03 & 333 & 474 \\
168.0 & & &
\end{tabular}

Table 25.--Concentrations of the four trihalomethane compounds and the total trihalomethane and nonpurgeable total organic-halide concentrations as a function of reaction time for an initial pH of 7.50 and an initial free-chlorine concentration of 30.0 milligrams per liter, Mississippi River at Natchez, Miss., March 26, 1992

$\left[\mu \mathrm{g} / \mathrm{L}\right.$, microgram per liter; $\mathrm{CHCl}_{3}$, chloroform; $\mathrm{BrCHCl}_{2}$, bromodichloromethane; $\mathrm{ClCHBr}_{2}$, chlorodibromomethane; $\mathrm{CHBr}_{3}$, bromoform; $\mathrm{THM}$, trihalomethane; NPTOX, nonpurgeable total organic halide]

\begin{tabular}{ccccccc}
\hline $\begin{array}{c}\text { Reaction } \\
\text { time } \\
\text { (hours) }\end{array}$ & $\mathrm{CHCl}_{3}$ & $\mathrm{BrCHCl}_{2}$ & $\mathrm{ClCHBr}_{2}$ & $\mathbf{C H B r}_{3}$ & Totai THM & NPTOX \\
\cline { 2 - 7 } & 19.9 & 2.49 & 0.14 & $\mathrm{ND}$ & 22.5 & 88.0 \\
0.00 & 74.8 & 12.6 & 1.29 & .02 & 88.7 & 225 \\
2.00 & 101 & 16.6 & 1.71 & .02 & 119 & 296 \\
6.00 & 125 & 17.6 & 1.88 & .02 & 144 & 333 \\
12.00 & 158 & 20.5 & 2.03 & .02 & 181 & 382 \\
24.00 & & & & & & \\
& 199 & 22.5 & 2.21 & .02 & 224 & 424 \\
48.00 & 229 & 22.7 & 2.12 & .02 & 254 & 426 \\
72.00 & 272 & 25.3 & 2.26 & ND & 300 & 455 \\
125.0 & 313 & 26.7 & 2.34 & .02 & 342 & 472 \\
168.0 & 318 & 27.1 & 2.33 & ND & 347 & 481 \\
168.0 & & & &
\end{tabular}


Tabie 26.--Concentrations of the four trihaiomethane compounds and the total trihalomethane and nonpurgeable total organic-halide-concentrations as a function of reaction time for an initiai pH of 7.50 and an initial free-chiorine concentration of 30.0 miliigrams per iiter, Mississippi River at Baton Rouge, La., March 26, 1992

$\left[\mu \mathrm{g} / \mathrm{L}\right.$, microgram per liter; $\mathrm{CHCl}_{3}$, chloroform; $\mathrm{BrCHCl}_{2}$, bromodichloromethane; $\mathrm{ClCHBr}_{2}$, chlorodibromomethane; $\mathrm{CHBr}_{3}$, bromoform; $\mathrm{THM}$, trihalomethane; NPTOX, nonpurgeable total organic halide]

\begin{tabular}{ccccccc}
\hline $\begin{array}{c}\text { Reaction } \\
\text { time } \\
\text { (hours) }\end{array}$ & CHCi $_{3}$ & BrCHCi $_{2}$ & ClCHBr $_{2}$ & CHBr $_{3}$ & Totai THM & NPTOX \\
\hline 0.00 & 18.3 & 2.49 & 0.22 & ND & 21.0 & 82.3 \\
2.00 & 74.5 & 13.4 & 1.06 & ND & 89.0 & 210 \\
6.00 & 95.3 & 16.0 & 1.60 & .02 & 113 & 298 \\
12.00 & 121 & 18.0 & 1.88 & .02 & 141 & 330 \\
24.00 & 154 & 20.0 & 2.00 & ND & 176 & 378 \\
& & & & & & \\
48.00 & 200 & 23.2 & 2.24 & .03 & 225 & 394 \\
72.00 & 237 & 23.7 & 2.20 & ND & 263 & 444 \\
126.0 & 285 & 25.8 & 2.32 & ND & 313 & 457 \\
168.0 & 345 & 25.8 & 2.34 & .01 & 373 & 472 \\
168.0 & 323 & 25.9 & 2.34 & ND & 351 & 449 \\
\hline
\end{tabular}

Table 27.--Concentrations of the four trihalomethane compounds and the total trihaiomethane and nonpurgeable total organic-hailde concentrations as a function of reaction time for an initiai $\mathrm{pH}$ of $\mathbf{7 . 5 0}$ and an initiai free-chiorine concentration of $\mathbf{3 0 . 0}$ milligrams per liter, Mississippi River at New Orleans, La., March 25, 1992

$\left[\mu \mathrm{g} / \mathrm{L}\right.$, microgram per liter; $\mathrm{CHCl}_{3}$, chloroform; $\mathrm{BrCHCl}_{2}$, bromodichloromethane; $\mathrm{ClCHBr}_{2}$, chlorodibromomethane; $\mathrm{CHBr}_{3}$, bromoform; $\mathrm{THM}$, trihalomethane; NPTOX, nonpurgeable total organic halide]

\begin{tabular}{ccccccc}
\hline $\begin{array}{c}\text { Reaction } \\
\text { time } \\
\text { (hours) }\end{array}$ & $\mathrm{CHCl}_{3}$ & $\mathrm{BrCHCl}_{2}$ & ClCHBr $_{2}$ & CHBr $_{3}$ & Total THM & NPTOX \\
\cline { 2 - 7 } & 15.8 & 3.74 & 0.77 & 0.02 & 20.3 & 71.6 \\
0.00 & 59.0 & 19.0 & 4.36 & .12 & 82.5 & 180 \\
2.03 & 79.1 & 24.1 & 5.43 & .15 & 109 & 245 \\
6.00 & 102 & 28.4 & 6.44 & .16 & 137 & 296 \\
12.00 & 122 & 29.8 & 6.66 & .17 & 159 & 323 \\
24.00 & & & & & & \\
& 159 & 33.8 & 7.15 & .19 & 200 & 372 \\
48.00 & 185 & 35.9 & 7.39 & .18 & 228 & 395 \\
72.00 & 220 & 39.4 & 7.61 & .20 & 267 & 396 \\
120.5 & 245 & 40.3 & 7.45 & .19 & 293 & 401 \\
168.0 & 254 & 40.9 & 7.54 & .19 & 303 & 401 \\
168.0 & & & & & & \\
\hline
\end{tabular}




\section{Ancillary Data}

\section{Values of $\mathrm{pH}$ as a Function of Reaction Time}

Values of $\mathrm{pH}$ as a function of reaction time for the different combinations of initial $\mathrm{pH}$ and initial free-chlorine concentration are listed in tables 28-41.

Table 28.--Variation of $\mathrm{pH}$ with reaction time for different combinations of initial $\mathrm{pH}$ and initial free-chlorine concentration, Mississippi River at Minneapolis, Minn.,

April 6, 1992

[CL, initial free-chlorine concentration; $\mathrm{mg} / \mathrm{L}$, milligram per liter]

\begin{tabular}{|c|c|c|c|c|c|}
\hline \multicolumn{2}{|c|}{$C L=15.0 \mathrm{mg} / \mathrm{L}$} & \multicolumn{2}{|c|}{$C L=30.0 \mathrm{mg} / \mathrm{L}$} & \multicolumn{2}{|c|}{$C L=50.0 \mathrm{mg} / \mathrm{L}$} \\
\hline $\begin{array}{l}\text { Reaction } \\
\text { time } \\
\text { (hours) }\end{array}$ & $\mathbf{p H}$ & $\begin{array}{c}\text { Reaction } \\
\text { time } \\
\text { (hours) }\end{array}$ & $\mathrm{pH}$ & $\begin{array}{c}\text { Reaction } \\
\text { time } \\
\text { (hours) }\end{array}$ & $\mathrm{pH}$ \\
\hline \multicolumn{6}{|c|}{ Initial $\mathrm{pH}=\mathbf{5 . 5 0}$} \\
\hline 0.00 & 5.50 & 0.00 & 5.50 & 0.00 & 5.50 \\
\hline 1.68 & 6.48 & 1.42 & 6.62 & 1.23 & 6.88 \\
\hline 26.02 & 6.44 & 25.77 & 6.62 & 25.60 & 6.77 \\
\hline 54.32 & 6.48 & 53.97 & 6.69 & 53.80 & 6.79 \\
\hline 78.62 & 6.51 & 78.37 & 6.77 & 78.20 & 6.78 \\
\hline 102.7 & 6.57 & 102.4 & 6.84 & 102.3 & 6.82 \\
\hline 126.8 & 6.61 & 126.6 & 6.89 & 126.4 & 6.82 \\
\hline 170.4 & 6.69 & 170.2 & 7.07 & 170.0 & 6.87 \\
\hline \multicolumn{6}{|c|}{ Initial pH $=7.50$} \\
\hline .00 & 7.50 & .00 & 7.50 & .00 & 7.50 \\
\hline 2.42 & 8.19 & 2.25 & 8.38 & 2.08 & 8.57 \\
\hline 26.80 & 8.09 & 26.63 & 8.23 & 26.47 & 8.43 \\
\hline 54.98 & 8.12 & 54.82 & 8.19 & 54.63 & 8.39 \\
\hline 79.40 & 8.13 & 79.23 & 8.13 & 79.07 & 8.36 \\
\hline 103.5 & 8.19 & 103.3 & 8.14 & 103.1 & 8.34 \\
\hline 127.6 & 8.22 & 127.5 & 8.13 & 127.3 & 8.32 \\
\hline 171.2 & 8.29 & 171.0 & 8.13 & 170.8 & 8.31 \\
\hline \multicolumn{6}{|c|}{ Initial pH $=10.00$} \\
\hline .00 & 10.00 & .00 & 10.00 & .00 & 10.00 \\
\hline .53 & 9.91 & .37 & 9.95 & .22 & 9.98 \\
\hline 24.92 & 9.78 & 24.75 & 9.83 & 24.60 & 9.86 \\
\hline 53.08 & 9.77 & 52.90 & 9.81 & 52.73 & 9.84 \\
\hline 77.52 & 9.74 & 77.35 & 9.78 & 77.20 & 9.81 \\
\hline 101.6 & 9.74 & 101.4 & 9.79 & 101.2 & 9.82 \\
\hline 125.8 & 9.70 & 125.6 & 9.77 & 125.4 & 9.79 \\
\hline 169.3 & 9.67 & 169.1 & 9.75 & 169.0 & 9.77 \\
\hline
\end{tabular}


Table 29.--Variation of $\mathrm{pH}$ with reaction time for different combinations of initial $\mathrm{pH}$ and initial free-chlorine concentration, Mississippi River at LaCrosse, Wis., April 3, 1992

$[\mathrm{CL}$, initial free-chlorine concentration; $\mathrm{mg} / \mathrm{L}$, milligram per liter]

\begin{tabular}{|c|c|c|c|c|c|}
\hline \multicolumn{2}{|c|}{$C L=15.0 \mathrm{mg} / \mathrm{L}$} & \multicolumn{2}{|c|}{$C L=30.0 \mathrm{mg} / \mathrm{L}$} & \multicolumn{2}{|c|}{$C L=50.0 \mathrm{mg} / \mathrm{L}$} \\
\hline $\begin{array}{c}\text { Reaction } \\
\text { tlme } \\
\text { (hours) }\end{array}$ & pH & $\begin{array}{c}\text { Reaction } \\
\text { time } \\
\text { (hours) }\end{array}$ & pH & $\begin{array}{c}\text { Reaction } \\
\text { time } \\
\text { (hours) }\end{array}$ & $\mathbf{p H}$ \\
\hline \multicolumn{6}{|c|}{ Initial pH $=\mathbf{5 . 5 0}$} \\
\hline 0.00 & 5.50 & 0.00 & 5.50 & 0.00 & 5.50 \\
\hline 1.77 & 6.63 & 1.62 & 6.67 & 1.47 & 6.90 \\
\hline 29.18 & 6.61 & 29.03 & 6.62 & 28.88 & 6.80 \\
\hline 54.10 & 6.64 & 53.95 & 6.60 & 53.80 & 6.78 \\
\hline 78.02 & 6.70 & 77.87 & 6.66 & 77.72 & 6.83 \\
\hline 125.3 & 6.74 & 125.1 & 6.70 & 125.0 & 6.85 \\
\hline 170.0 & 6.82 & 169.9 & 6.76 & 169.7 & 6.87 \\
\hline \multicolumn{6}{|c|}{ Initial pH = 7.50} \\
\hline .00 & 7.50 & .00 & 7.50 & .00 & 7.50 \\
\hline 2.62 & 8.25 & 2.30 & 8.24 & 2.40 & 8.55 \\
\hline 30.00 & 8.15 & 29.68 & 8.10 & 29.80 & 8.47 \\
\hline 54.95 & 8.09 & 54.62 & 8.05 & 54.73 & 8.41 \\
\hline 78.83 & 8.11 & 78.52 & 8.09 & 78.63 & 8.41 \\
\hline 126.1 & 8.11 & 125.8 & 8.12 & 125.9 & 8.42 \\
\hline 170.9 & 8.14 & 170.6 & 8.11 & 170.7 & 8.40 \\
\hline \multicolumn{6}{|c|}{ Initial pH $=10.00$} \\
\hline .00 & 10.00 & .00 & 10.00 & .00 & 10.00 \\
\hline .80 & 9.66 & .65 & 9.80 & .50 & 9.83 \\
\hline 28.18 & 9.54 & 28.03 & 9.69 & 27.88 & 9.73 \\
\hline 53.12 & 9.49 & 52.97 & 9.64 & 52.82 & 9.69 \\
\hline 77.03 & 9.49 & 76.88 & 9.64 & 76.70 & 9.68 \\
\hline 124.3 & 9.45 & 124.2 & 9.60 & 124.0 & 9.66 \\
\hline 169.1 & 9.42 & 168.9 & 9.57 & 168.8 & 9.64 \\
\hline
\end{tabular}


Table 30.-Variation of pH with reaction time for different combinations of initial pH and initial free-chlorine concentration, Mississippi River at Dubuque, lowa, April 2, 1992

[CL, initial free-chlorine concentration; $\mathrm{mg} / \mathrm{L}$, milligram per liter]

\begin{tabular}{|c|c|c|c|c|c|}
\hline \multicolumn{2}{|c|}{$\mathrm{CL}=15.0 \mathrm{mg} / \mathrm{L}$} & \multicolumn{2}{|c|}{$C L=30.0 \mathrm{mg} / \mathrm{L}$} & \multicolumn{2}{|c|}{$C L=50.0 \mathrm{mg} / \mathrm{L}$} \\
\hline $\begin{array}{c}\text { Reaction } \\
\text { time } \\
\text { (hours) }\end{array}$ & pH & $\begin{array}{c}\text { Reaction } \\
\text { time } \\
\text { (hours) }\end{array}$ & pH & $\begin{array}{c}\text { Reaction } \\
\text { time } \\
\text { (hours) }\end{array}$ & pH \\
\hline \multicolumn{6}{|c|}{ Initial $\mathrm{pH}=\mathbf{5 . 5 0}$} \\
\hline 0.00 & 5.50 & 0.00 & 5.50 & 0.00 & 5.50 \\
\hline 1.80 & 6.77 & 1.65 & 6.82 & 1.48 & 7.18 \\
\hline 24.00 & 6.65 & 23.85 & 6.71 & 23.68 & 7.04 \\
\hline 47.98 & 6.64 & 47.83 & 6.71 & 47.67 & 7.03 \\
\hline 71.88 & 6.65 & 71.73 & 6.75 & 71.57 & 7.12 \\
\hline 120.2 & 6.60 & 120.1 & 6.74 & 119.9 & 7.16 \\
\hline 170.3 & 6.68 & 170.1 & 6.82 & 169.9 & 7.33 \\
\hline \multicolumn{6}{|c|}{ Initial pH = $\mathbf{7 . 5 0}$} \\
\hline .00 & 7.50 & .00 & 7.50 & .00 & 7.50 \\
\hline 2.68 & 8.32 & 2.35 & 8.37 & 2.50 & 8.65 \\
\hline 24.90 & 8.16 & 24.57 & 8.20 & 24.72 & 8.52 \\
\hline 48.88 & 8.14 & 48.53 & 8.16 & 48.68 & 8.49 \\
\hline 72.78 & 8.13 & 72.45 & 8.13 & 72.60 & 8.47 \\
\hline 121.1 & 8.06 & 120.8 & 8.06 & 121.0 & 8.40 \\
\hline 171.2 & 8.09 & 170.8 & 8.08 & 171.0 & 8.39 \\
\hline \multicolumn{6}{|c|}{ Initial $\mathbf{p H}=\mathbf{1 0 . 0 0}$} \\
\hline .00 & 10.00 & .00 & 10.00 & .00 & 10.00 \\
\hline .70 & 9.89 & .57 & 9.97 & .40 & 10.00 \\
\hline 22.95 & 9.65 & 22.82 & 9.75 & 22.67 & 9.78 \\
\hline 46.93 & 9.62 & 46.80 & 9.72 & 46.65 & 9.75 \\
\hline 70.85 & 9.60 & 70.72 & 9.70 & 70.57 & 9.74 \\
\hline 119.2 & 9.53 & 119.1 & 9.63 & 118.9 & 9.67 \\
\hline 169.2 & 9.53 & 169.1 & 9.63 & 168.9 & 9.64 \\
\hline
\end{tabular}


Tabie 31.-Variation of pH with reaction time for different combinations of Initial pH and initiai free-chiorine concentration, Mississippi River at Davenport, lowa, Aprii 2, 1992

[CL, initial free-chlorine concentration; $\mathrm{mg} / \mathrm{L}$, milligram per liter; $\mathrm{NS}$, no sample because of insufficient water]

\begin{tabular}{|c|c|c|c|c|c|}
\hline \multicolumn{2}{|c|}{$C L=15.0 \mathrm{mg} / \mathrm{L}$} & \multicolumn{2}{|c|}{$\mathrm{CL}=30.0 \mathrm{mg} / \mathrm{L}$} & \multicolumn{2}{|c|}{$\mathrm{CL}=50.0 \mathrm{mg} / \mathrm{L}$} \\
\hline $\begin{array}{c}\text { Reaction } \\
\text { time } \\
\text { (hours) }\end{array}$ & $\mathrm{pH}$ & $\begin{array}{c}\text { Reaction } \\
\text { time } \\
\text { (hours) }\end{array}$ & pH & $\begin{array}{c}\text { Reaction } \\
\text { time } \\
\text { (hours) }\end{array}$ & $\mathrm{pH}$ \\
\hline \multicolumn{6}{|c|}{ Initial $\mathbf{p H}=5.50$ (regular sample) } \\
\hline NS & NS & 0.00 & 5.50 & 0.00 & 5.50 \\
\hline NS & NS & 1.95 & 6.70 & 1.77 & 6.97 \\
\hline NS & NS & 30.75 & 6.65 & 30.55 & 6.81 \\
\hline NS & NS & 52.62 & 6.70 & 52.38 & 6.82 \\
\hline NS & NS & 76.55 & 6.80 & 76.35 & 6.83 \\
\hline NS & NS & 123.4 & 6.91 & 123.2 & 6.88 \\
\hline NS & NS & 172.4 & 7.15 & 172.2 & 6.89 \\
\hline \multicolumn{6}{|c|}{ Initial pH $=7.50$ (regular sample) } \\
\hline NS & NS & .00 & 7.50 & .00 & 7.50 \\
\hline NS & NS & 2.97 & 8.38 & 2.78 & 8.59 \\
\hline NS & NS & 31.82 & 8.21 & 31.57 & 8.46 \\
\hline NS & NS & 53.67 & 8.17 & 53.42 & 8.42 \\
\hline NS & NS & 77.60 & 8.13 & 77.42 & 8.40 \\
\hline NS & NS & 124.4 & 8.12 & 124.2 & 8.39 \\
\hline NS & NS & 173.4 & 8.10 & 173.2 & 8.38 \\
\hline \multicolumn{6}{|c|}{ Initial $\mathrm{pH}=10.00$ (regular sample) } \\
\hline 0.00 & 10.00 & .00 & 10.00 & .00 & 10.00 \\
\hline .58 & 9.90 & .42 & 9.93 & .22 & 9.97 \\
\hline 29.38 & 9.64 & 29.22 & 9.66 & 29.02 & 9.71 \\
\hline 51.22 & 9.61 & 51.05 & 9.64 & 50.83 & 9.69 \\
\hline 75.22 & 9.58 & 75.05 & 9.61 & 74.85 & 9.67 \\
\hline 122.1 & 9.55 & 121.9 & 9.59 & 121.7 & 9.65 \\
\hline 171.0 & 9.51 & 170.8 & 9.54 & 170.6 & 9.63 \\
\hline
\end{tabular}


Table 31.-Variation of pH with reaction time for different combinations of initial pH and initial free-chlorine concentration, Mississippi River at Davenport, lowa, April 2, 1992-Continued

\begin{tabular}{|c|c|c|c|c|c|}
\hline \multicolumn{2}{|c|}{$C L=15.0 \mathrm{mg} / \mathrm{L}$} & \multicolumn{2}{|c|}{$C L=30.0 \mathrm{mg} / \mathrm{L}$} & \multicolumn{2}{|c|}{$C L=50.0 \mathrm{mg} / \mathrm{L}$} \\
\hline $\begin{array}{l}\text { Reaction } \\
\text { time } \\
\text { (hours) }\end{array}$ & $\mathbf{p H}$ & $\begin{array}{l}\text { Reaction } \\
\text { time } \\
\text { (hours) }\end{array}$ & pH & $\begin{array}{c}\text { Reaction } \\
\text { time } \\
\text { (hours) }\end{array}$ & pH \\
\hline \multicolumn{6}{|c|}{ Initial pH $=5.50$ (thawed sample) } \\
\hline NS & NS & 0.00 & 5.50 & 0.00 & 5.50 \\
\hline NS & NS & 2.50 & 6.70 & 2.32 & 6.93 \\
\hline NS & NS & 31.37 & 6.63 & 31.18 & 7.01 \\
\hline NS & NS & 53.17 & 6.68 & 53.02 & 7.17 \\
\hline NS & NS & 77.17 & 6.81 & 76.98 & 7.31 \\
\hline NS & NS & 124.0 & 6.98 & 123.8 & 7.56 \\
\hline NS & NS & 173.0 & 7.08 & 172.8 & 7.70 \\
\hline \multicolumn{6}{|c|}{ Initial $\mathrm{pH}=7.50$ (thawed sample) } \\
\hline NS & NS & .00 & 7.50 & .00 & 7.50 \\
\hline NS & NS & 3.53 & 8.39 & 3.30 & 8.61 \\
\hline NS & NS & 32.32 & 8.22 & 32.15 & 8.48 \\
\hline NS & NS & 54.13 & 8.19 & 53.93 & 8.45 \\
\hline NS & NS & 78.17 & 8.16 & 77.93 & 8.44 \\
\hline NS & NS & 125.0 & 8.16 & 124.8 & 8.44 \\
\hline NS & NS & 174.0 & 8.16 & 173.8 & 8.44 \\
\hline \multicolumn{6}{|c|}{ Initial $\mathrm{pH}=10.00$ (thawed sample) } \\
\hline NS & NS & .00 & 10.00 & .00 & 10.00 \\
\hline NS & NS & 1.07 & 9.92 & .87 & 9.94 \\
\hline NS & NS & 29.87 & 9.73 & 29.67 & 9.77 \\
\hline NS & NS & 51.70 & 9.71 & 51.48 & 9.76 \\
\hline NS & NS & 75.70 & 9.68 & 75.50 & 9.73 \\
\hline NS & NS & 122.6 & 9.66 & 122.4 & 9.72 \\
\hline NS & NS & 171.6 & 9.63 & 171.4 & 9.69 \\
\hline
\end{tabular}


Tabie 32.-Variation of $\mathrm{pH}$ with reaction time for different combinations of initiai $\mathrm{pH}$ and initiai free-chlorine concentration, Mississippi River at Quincy, lli., April 1, 1992

[CL, initial free-chlorine concentration; $\mathrm{mg} / \mathrm{L}$, milligram per liter]

\begin{tabular}{|c|c|c|c|c|c|}
\hline \multicolumn{2}{|c|}{$C L=15.0 \mathrm{mg} / \mathrm{L}$} & \multicolumn{2}{|c|}{$\mathrm{CL}=30.0 \mathrm{mg} / \mathrm{L}$} & \multicolumn{2}{|c|}{$C L=50.0 \mathrm{mg} / \mathrm{L}$} \\
\hline $\begin{array}{c}\text { Reaction } \\
\text { time } \\
\text { (hours) }\end{array}$ & pH & $\begin{array}{c}\text { Reaction } \\
\text { time } \\
\text { (hours) }\end{array}$ & pH & $\begin{array}{c}\text { Reaction } \\
\text { time } \\
\text { (hours) }\end{array}$ & pH \\
\hline \multicolumn{6}{|c|}{ Initial pH $=\mathbf{5 . 5 0}$} \\
\hline 0.00 & 5.50 & 0.00 & 5.50 & 0.00 & 5.50 \\
\hline 1.73 & 6.71 & 1.57 & 6.74 & 1.40 & 6.99 \\
\hline 31.22 & 6.63 & 31.05 & 6.71 & 30.88 & 6.87 \\
\hline 54.17 & 6.67 & 54.00 & 6.83 & 53.83 & 6.92 \\
\hline 79.00 & 6.74 & 78.83 & 7.08 & 78.67 & 7.01 \\
\hline 119.4 & 7.04 & 119.3 & 7.40 & 119.1 & 7.05 \\
\hline 170.2 & 7.16 & 170.0 & 7.56 & 169.9 & 7.11 \\
\hline \multicolumn{6}{|c|}{ Initial pH = 7.50} \\
\hline .00 & 7.50 & .00 & 7.50 & .00 & 7.50 \\
\hline 2.70 & 8.28 & 2.30 & 8.16 & 2.50 & 8.53 \\
\hline 32.18 & 8.15 & 31.78 & 8.03 & 31.97 & 8.42 \\
\hline 55.12 & 8.15 & 54.72 & 8.04 & 54.92 & 8.40 \\
\hline 79.97 & 8.18 & 79.57 & 8.03 & 79.77 & 8.38 \\
\hline 120.4 & 8.21 & 120.0 & 8.06 & 120.2 & 8.38 \\
\hline 171.2 & 8.20 & 170.8 & 8.09 & 171.0 & 8.36 \\
\hline \multicolumn{6}{|c|}{ Initial $\mathbf{p H}=10.00$} \\
\hline .00 & 10.00 & .00 & 10.00 & .00 & 10.00 \\
\hline .75 & 9.74 & .58 & 9.79 & .43 & 9.81 \\
\hline 30.22 & 9.64 & 30.05 & 9.70 & 29.90 & 9.73 \\
\hline 53.17 & 9.60 & 53.00 & 9.66 & 52.85 & 9.71 \\
\hline 78.02 & 9.58 & 77.85 & 9.64 & 77.70 & 9.70 \\
\hline 118.4 & 9.57 & 118.3 & 9.61 & 118.1 & 9.69 \\
\hline 169.2 & 9.53 & 169.1 & 9.57 & 168.9 & 9.66 \\
\hline
\end{tabular}


Table 33.-Variation of $\mathrm{pH}$ with reaction time for different combinations of initial $\mathrm{pH}$ and initial free-chlorine concentration, Missouri River 1.6 kilometers upstream from confluence with Mississippi River, March 31, 1992

[CL, initial free-chlorine concentration; $\mathrm{mg} / \mathrm{L}$, milligram per liter]

\begin{tabular}{|c|c|c|c|c|c|}
\hline \multicolumn{2}{|c|}{$C L=15.0 \mathrm{mg} / \mathrm{L}$} & \multicolumn{2}{|c|}{$\mathrm{CL}=30.0 \mathrm{mg} / \mathrm{L}$} & \multicolumn{2}{|c|}{$C L=50.0 \mathrm{mg} / \mathrm{L}$} \\
\hline $\begin{array}{l}\text { Reaction } \\
\text { time } \\
\text { (hours) }\end{array}$ & pH & $\begin{array}{c}\text { Reaction } \\
\text { time } \\
\text { (hours) }\end{array}$ & pH & $\begin{array}{c}\text { Reaction } \\
\text { time } \\
\text { (hours) }\end{array}$ & pH \\
\hline \multicolumn{6}{|c|}{ Initial pH $=5.50$} \\
\hline 0.00 & 5.50 & 0.00 & 5.50 & 0.00 & 5.50 \\
\hline 1.72 & 6.70 & 1.53 & 6.76 & 1.35 & 7.05 \\
\hline 22.33 & 6.66 & 22.17 & 6.71 & 21.98 & 6.97 \\
\hline 48.78 & 6.83 & 48.62 & 6.79 & 48.42 & 7.07 \\
\hline 70.78 & 6.87 & 70.62 & 6.84 & 70.43 & 7.19 \\
\hline 119.9 & 6.93 & 119.8 & 6.91 & 119.6 & 7.36 \\
\hline 170.3 & 6.96 & 170.2 & 7.03 & 170.0 & 7.56 \\
\hline \multicolumn{6}{|c|}{ Initial pH = $\mathbf{7 . 5 0}$} \\
\hline .00 & 7.50 & .00 & 7.50 & .00 & 7.50 \\
\hline 2.65 & 8.31 & 2.32 & 8.31 & 2.45 & 8.59 \\
\hline 23.25 & 8.22 & 22.92 & 8.22 & 23.03 & 8.53 \\
\hline 49.72 & 8.21 & 49.38 & 8.20 & 49.50 & 8.50 \\
\hline 71.75 & 8.22 & 71.40 & 8.19 & 71.52 & 8.49 \\
\hline 120.9 & 8.26 & 120.5 & 8.21 & 120.7 & 8.47 \\
\hline 171.2 & 8.31 & 170.9 & 8.20 & 171.0 & 8.47 \\
\hline \multicolumn{6}{|c|}{ Imitial $\mathbf{p H}=\mathbf{1 0 . 0 0}$} \\
\hline .00 & 10.00 & .00 & 10.00 & .00 & 10.00 \\
\hline .67 & 9.81 & .50 & 9.85 & .33 & 9.87 \\
\hline 21.27 & 9.64 & 21.10 & 9.72 & 20.93 & 9.75 \\
\hline 47.73 & 9.58 & 47.57 & 9.68 & 47.40 & 9.72 \\
\hline 69.75 & 9.53 & 69.58 & 9.65 & 69.42 & 9.69 \\
\hline 118.9 & 9.41 & 118.7 & 9.63 & 118.6 & 9.67 \\
\hline 169.3 & 9.35 & 169.1 & 9.61 & 168.9 & 9.66 \\
\hline
\end{tabular}


Table 34.-Variation of $\mathrm{pH}$ with reaction time for different combinations of initial $\mathrm{pH}$ and Initial free-chlorine concentration, Mississippi River at St. Louis, Mo., March 31, 1992

[CL, initial free-chlorine concentration; $\mathrm{mg} / \mathrm{L}$, milligram per liter]

\begin{tabular}{|c|c|c|c|c|c|}
\hline \multicolumn{2}{|c|}{$C L=15.0 \mathrm{mg} / \mathrm{L}$} & \multicolumn{2}{|c|}{$C L=30.0 \mathrm{mg} / \mathrm{L}$} & \multicolumn{2}{|c|}{$C L=50.0 \mathrm{mg} / \mathrm{L}$} \\
\hline $\begin{array}{c}\text { Reaction } \\
\text { time } \\
\text { (hours) }\end{array}$ & pH & $\begin{array}{c}\text { Reaction } \\
\text { time } \\
\text { (hours) }\end{array}$ & pH & $\begin{array}{c}\text { Reaction } \\
\text { time } \\
\text { (hours) }\end{array}$ & pH \\
\hline \multicolumn{6}{|c|}{ Initial $\mathbf{p H}=\mathbf{5 . 5 0}$} \\
\hline 0.00 & 5.50 & 0.00 & 5.50 & 0.00 & 5.50 \\
\hline 1.68 & 6.72 & 1.52 & 6.78 & 1.35 & 7.03 \\
\hline 23.80 & 6.66 & 23.63 & 6.77 & 23.47 & 6.96 \\
\hline 47.85 & 6.72 & 47.68 & 6.92 & .47 .52 & 7.04 \\
\hline 71.78 & 6.79 & 71.62 & 6.99 & 71.45 & 7.08 \\
\hline 120.3 & 6.89 & 120.1 & 7.22 & 119.9 & 7.27 \\
\hline 170.3 & 6.96 & 170.1 & 7.34 & 170.0 & 7.43 \\
\hline \multicolumn{6}{|c|}{ Initial $\mathbf{p H}=\mathbf{7 . 5 0}$} \\
\hline .00 & 7.50 & .00 & 7.50 & .00 & 7.50 \\
\hline 2.60 & 8.39 & 2.28 & 8.29 & 2.40 & 8.61 \\
\hline 24.72 & 8.27 & 24.40 & 8.20 & 24.52 & 8.51 \\
\hline 48.77 & 8.24 & 48.43 & 8.19 & 48.55 & 8.48 \\
\hline 72.70 & 8.26 & 72.38 & 8.21 & 72.50 & 8.46 \\
\hline 121.2 & 8.29 & 120.8 & 8.29 & 121.0 & 8.45 \\
\hline 171.2 & 8.31 & 170.9 & 8.36 & 171.0 & 8.45 \\
\hline \multicolumn{6}{|c|}{ Initial $\mathrm{pH}=10.00$} \\
\hline .00 & 10.00 & .00 & 10.00 & .00 & 10.00 \\
\hline .67 & 9.90 & .50 & 9.93 & .35 & 9.94 \\
\hline 22.80 & 9.71 & 22.63 & 9.76 & 22.48 & 9.80 \\
\hline 46.83 & 9.67 & 46.67 & 9.72 & 46.52 & 9.77 \\
\hline 70.77 & 9.66 & 70.60 & 9.71 & 70.45 & 9.76 \\
\hline 119.2 & 9.62 & 119.1 & 9.69 & 118.9 & 9.75 \\
\hline 169.3 & 9.60 & 169.1 & 9.68 & 169.0 & 9.73 \\
\hline
\end{tabular}


Table 35.--Variation of $\mathrm{pH}$ with reaction time for different combinations of initial $\mathrm{pH}$ and initial free-chlorine concentration, Mississippi River at Cairo, ill., March 30, 1992

[CL, initial free-chlorine concentration; $\mathrm{mg} / \mathrm{L}$, milligram per liter]

\begin{tabular}{|c|c|c|c|c|c|}
\hline \multicolumn{2}{|c|}{$C L=15.0 \mathrm{mg} / \mathrm{L}$} & \multicolumn{2}{|c|}{$\mathrm{CL}=30.0 \mathrm{mg} / \mathrm{L}$} & \multicolumn{2}{|c|}{$\mathrm{CL}=50.0 \mathrm{mg} / \mathrm{L}$} \\
\hline $\begin{array}{c}\text { Reaction } \\
\text { time } \\
\text { (hours) }\end{array}$ & pH & $\begin{array}{c}\text { Reaction } \\
\text { time } \\
\text { (hours) }\end{array}$ & pH & $\begin{array}{c}\text { Reaction } \\
\text { time } \\
\text { (hours) }\end{array}$ & pH \\
\hline \multicolumn{6}{|c|}{ Initial pH $=\mathbf{5 . 5 0}$} \\
\hline 0.00 & 5.50 & 0.00 & 5.50 & 0.00 & 5.50 \\
\hline 1.73 & 6.71 & 1.57 & 6.74 & 1.42 & 7.00 \\
\hline 25.30 & 6.65 & 25.12 & 6.67 & 24.95 & 6.92 \\
\hline 53.95 & 6.76 & 53.75 & 6.77 & 53.60 & 6.95 \\
\hline 78.68 & 6.79 & 78.52 & 6.82 & 78.37 & 6.97 \\
\hline 125.2 & 7.09 & 125.0 & 7.00 & 124.8 & 7.07 \\
\hline 170.3 & 7.21 & 170.1 & 7.20 & 170.0 & 7.10 \\
\hline \multicolumn{6}{|c|}{ Initial pH = $\mathbf{7 . 5 0}$} \\
\hline .00 & 7.50 & .00 & 7.50 & .00 & 7.50 \\
\hline 2.68 & 8.28 & 2.35 & 8.29 & 2.48 & 8.57 \\
\hline 26.22 & 8.20 & 25.88 & 8.20 & 25.98 & 8.50 \\
\hline 54.87 & 8.14 & 54.53 & 8.15 & 54.67 & 8.46 \\
\hline 79.62 & 8.16 & 79.28 & 8.16 & 79.38 & 8.47 \\
\hline 126.1 & 8.25 & 125.8 & 8.18 & 125.9 & 8.45 \\
\hline 171.2 & 8.31 & 170.9 & 8.22 & 171.0 & 8.45 \\
\hline \multicolumn{6}{|c|}{ Initial $\mathbf{p H}=\mathbf{1 0 . 0 0}$} \\
\hline .00 & 10.00 & .00 & 10.00 & .00 & 10.00 \\
\hline .73 & 9.78 & .57 & 9.83 & .42 & 9.85 \\
\hline 24.23 & 9.63 & 24.07 & 9.69 & 23.90 & 9.73 \\
\hline 52.92 & 9.56 & 52.73 & 9.64 & 52.58 & 9.68 \\
\hline 77.63 & 9.56 & 77.47 & 9.64 & 77.32 & 9.67 \\
\hline 124.2 & 9.51 & 124.0 & 9.60 & 123.8 & 9.64 \\
\hline 169.3 & 9.49 & 169.1 & 9.58 & 169.0 & 9.62 \\
\hline
\end{tabular}


Table 36.-Variation of $\mathrm{pH}$ with reaction time for different combinations of initial $\mathrm{pH}$ and initial free-chlorine concentration, Ohio River 1.6 kilometers upstream from confiuence with Mississippi River, March 30, 1992

[CL, initial free-chlorine concentration; $\mathrm{mg} / \mathrm{L}$, milligram per liter]

\begin{tabular}{|c|c|c|c|c|c|}
\hline \multicolumn{2}{|c|}{$C L=15.0 \mathrm{mg} / \mathrm{L}$} & \multicolumn{2}{|c|}{$C L=30.0 \mathrm{mg} / \mathrm{L}$} & \multicolumn{2}{|c|}{$\mathrm{CL}=50.0 \mathrm{mg} / \mathrm{L}$} \\
\hline $\begin{array}{c}\text { Reaction } \\
\text { time } \\
\text { (hours) }\end{array}$ & pH & $\begin{array}{c}\text { Reaction } \\
\text { time } \\
\text { (hours) }\end{array}$ & pH & $\begin{array}{c}\text { Reaction } \\
\text { time } \\
\text { (hours) }\end{array}$ & $\mathrm{pH}$ \\
\hline \multicolumn{6}{|c|}{ Initial pH $=5.50$} \\
\hline 0.00 & 5.50 & 0.00 & 5.50 & 0.00 & 5.50 \\
\hline 1.77 & 6.91 & 1.60 & 7.20 & 1.43 & 7.62 \\
\hline 25.70 & 6.85 & 25.53 & 7.12 & 25.37 & 7.53 \\
\hline 48.50 & 6.86 & 48.33 & 7.14 & 48.17 & 7.53 \\
\hline 71.92 & 6.89 & 71.75 & 7.17 & 71.57 & 7.54 \\
\hline 122.4 & 6.95 & 122.3 & 7.25 & 122.1 & 7.53 \\
\hline 170.4 & 6.96 & 170.2 & 7.29 & 170.0 & 7.55 \\
\hline \multicolumn{6}{|c|}{ Initial $\mathbf{p H}=\mathbf{7 . 5 0}$} \\
\hline .00 & 7.50 & .00 & 7.50 & .00 & 7.50 \\
\hline 2.65 & 8.49 & 2.32 & 8.67 & 2.45 & 8.91 \\
\hline 26.58 & 8.37 & 26.25 & 8.57 & 26.38 & 8.82 \\
\hline 49.37 & 8.32 & 49.03 & 8.55 & 49.15 & 8.80 \\
\hline 72.78 & 8.29 & 72.45 & 8.53 & 72.58 & 8.79 \\
\hline 123.3 & 8.24 & 122.9 & 8.47 & 123.1 & 8.73 \\
\hline 171.2 & 8.21 & 171.0 & 8.48 & 171.0 & 8.70 \\
\hline \multicolumn{6}{|c|}{ Initial pH = 10.00} \\
\hline .00 & 10.00 & .00 & 10.00 & .00 & 10.00 \\
\hline .68 & 9.97 & .52 & 10.02 & .32 & 10.04 \\
\hline 24.63 & 9.77 & 24.47 & 9.81 & 24.27 & 9.84 \\
\hline 47.40 & 9.69 & 47.23 & 9.74 & 47.03 & 9.77 \\
\hline 70.83 & 9.64 & 70.67 & 9.69 & 70.47 & 9.73 \\
\hline 121.4 & 9.59 & 121.2 & 9.65 & 121.0 & 9.70 \\
\hline 169.3 & 9.56 & 169.1 & 9.60 & 168.9 & 9.69 \\
\hline
\end{tabular}


Table 37.-Variation of $\mathrm{pH}$ with reaction time for different combinations of initial $\mathrm{pH}$ and initial free-chlorine concentration, Mississippi River at Memphis, Tenn., March 29, 1992

[CL, initial free-chlorine concentration; $\mathrm{mg} / \mathrm{L}$, milligram per liter]

\begin{tabular}{|c|c|c|c|c|c|}
\hline \multicolumn{2}{|c|}{$\mathrm{CL}=15.0 \mathrm{mg} / \mathrm{L}$} & \multicolumn{2}{|c|}{$C L=30.0 \mathrm{mg} / \mathrm{L}$} & \multicolumn{2}{|c|}{$C L=50.0 \mathrm{mg} / \mathrm{L}$} \\
\hline $\begin{array}{c}\text { Reaction } \\
\text { time } \\
\text { (hours) }\end{array}$ & pH & $\begin{array}{c}\text { Reaction } \\
\text { time } \\
\text { (hours) }\end{array}$ & pH & $\begin{array}{c}\text { Reaction } \\
\text { time } \\
\text { (hours) }\end{array}$ & pH \\
\hline \multicolumn{6}{|c|}{ Initial $\mathbf{p H}=\mathbf{5 . 5 0}$} \\
\hline 0.00 & 5.50 & 0.00 & 5.50 & 0.00 & 5.50 \\
\hline 1.72 & 6.75 & 1.53 & 6.90 & 1.37 & 7.27 \\
\hline 29.82 & 6.68 & 29.62 & 6.82 & 29.42 & 7.15 \\
\hline 55.42 & 6.71 & 55.23 & 6.93 & 55.07 & 7.18 \\
\hline 77.20 & 6.77 & 77.00 & 7.02 & 76.83 & 7.23 \\
\hline 125.1 & 6.86 & 124.9 & 7.05 & 124.8 & 7.31 \\
\hline 170.6 & 6.97 & 170.4 & 7.28 & 170.2 & 7.42 \\
\hline \multicolumn{6}{|c|}{ Initial $\mathbf{p H}=\mathbf{7 . 5 0}$} \\
\hline .00 & 7.50 & .00 & 7.50 & .00 & 7.50 \\
\hline 2.65 & 8.36 & 2.28 & 8.44 & 2.43 & 8.75 \\
\hline 30.68 & 8.24 & 30.28 & 8.66 & 30.43 & 8.64 \\
\hline 56.35 & 8.21 & 55.98 & 8.30 & 56.13 & 8.64 \\
\hline 78.12 & 8.20 & 77.75 & 8.29 & 77.90 & 8.62 \\
\hline 126.0 & 8.21 & 125.7 & 8.27 & 125.8 & 8.58 \\
\hline 171.5 & 8.24 & 171.2 & 8.27 & 171.3 & 8.56 \\
\hline \multicolumn{6}{|c|}{ Initial $\mathbf{p H}=\mathbf{1 0 . 0 0}$} \\
\hline .00 & 10.00 & .00 & 10.00 & .00 & 10.00 \\
\hline .68 & 9.95 & .52 & 10.00 & .35 & 10.03 \\
\hline 28.68 & 9.64 & 28.50 & 9.72 & 28.33 & 9.78 \\
\hline 54.38 & 9.53 & 54.22 & 9.66 & 54.05 & 9.72 \\
\hline 76.15 & 9.48 & 75.98 & 9.63 & 75.82 & 9.69 \\
\hline 124.1 & 9.34 & 123.9 & 9.58 & 123.7 & 9.66 \\
\hline 169.5 & 9.24 & 169.4 & 9.56 & 169.2 & 9.65 \\
\hline
\end{tabular}


Table 38.-Variation of $\mathrm{pH}$ wlth reaction time for different combinations of initial $\mathrm{pH}$ and Initial free-chlorine concentration, MississIppl River at Greenviile, Miss., March 28, 1992

[CL, initial free-chlorine concentration; $\mathrm{mg} / \mathrm{L}$, milligram per liter]

\begin{tabular}{|c|c|c|c|c|c|}
\hline \multicolumn{2}{|c|}{$C L=15.0 \mathrm{mg} / \mathrm{L}$} & \multicolumn{2}{|c|}{$C L=30.0 \mathrm{mg} / \mathrm{L}$} & \multicolumn{2}{|c|}{$\mathrm{CL}=50.0 \mathrm{mg} / \mathrm{L}$} \\
\hline $\begin{array}{l}\text { Reaction } \\
\text { time } \\
\text { (hours) }\end{array}$ & pH & $\begin{array}{c}\text { Reactlon } \\
\text { time } \\
\text { (hours) }\end{array}$ & pH & $\begin{array}{c}\text { Reaction } \\
\text { time } \\
\text { (hours) }\end{array}$ & pH \\
\hline \multicolumn{6}{|c|}{ Initial $\mathbf{p H}=\mathbf{5 . 5 0}$} \\
\hline 0.00 & 5.50 & 0.00 & 5.50 & 0.00 & 5.50 \\
\hline 1.43 & 6.55 & 1.35 & 6.80 & 1.25 & 7.20 \\
\hline 26.90 & 6.52 & 26.82 & 6.72 & 26.72 & 7.08 \\
\hline 54.12 & 6.60 & 53.97 & 6.75 & 53.80 & 7.12 \\
\hline 78.02 & 6.65 & 77.87 & 6.78 & 77.67 & 7.15 \\
\hline 121.2 & 6.71 & 121.0 & 7.02 & 120.8 & 7.19 \\
\hline 170.5 & 6.76 & 170.3 & 7.04 & 170.2 & 7.24 \\
\hline \multicolumn{6}{|c|}{ Initial $\mathbf{p H}=7.50$} \\
\hline .00 & 7.50 & .00 & 7.50 & .00 & 7.50 \\
\hline 2.53 & 8.44 & 2.27 & 8.56 & 2.48 & 8.80 \\
\hline 28.00 & 8.28 & 27.73 & 8.41 & 27.93 & 8.64 \\
\hline 55.02 & 8.26 & 54.68 & 8.39 & 54.80 & 8.63 \\
\hline 78.88 & 8.25 & 78.48 & 8.39 & 78.60 & 8.60 \\
\hline 122.1 & 8.26 & 121.7 & 8.40 & 121.9 & 8.58 \\
\hline 171.4 & 8.28 & 171.0 & 8.42 & 171.2 & 8.56 \\
\hline \multicolumn{6}{|c|}{ Initial $\mathbf{p H}=\mathbf{1 0 . 0 0}$} \\
\hline .00 & 10.00 & .00 & 10.00 & .00 & 10.00 \\
\hline .87 & 10.08 & .77 & 10.10 & .67 & 10.12 \\
\hline 26.32 & 9.74 & 26.22 & 9.75 & 26.10 & 9.81 \\
\hline 53.12 & 9.67 & 52.95 & 9.71 & 52.77 & 9.76 \\
\hline 76.92 & 9.64 & 76.72 & 9.69 & 76.53 & 9.74 \\
\hline 120.2 & 9.61 & 120.0 & 9.66 & 119.8 & 9.70 \\
\hline 169.5 & 9.57 & 169.3 & 9.63 & 169.1 & 9.68 \\
\hline
\end{tabular}


Table 39.--Variation of $\mathrm{pH}$ with reaction time for different combinations of initial $\mathrm{pH}$ and initial free-chlorine concentration, Mississippi River at Natchez, Miss., March 26, 1992

[CL, initial free-chlorine concentration; $\mathrm{mg} / \mathrm{L}$, milligram per liter]

\begin{tabular}{|c|c|c|c|c|c|}
\hline \multicolumn{2}{|c|}{$C L=15.0 \mathrm{mg} / \mathrm{L}$} & \multicolumn{2}{|c|}{$C L=30.0 \mathrm{mg} / \mathrm{L}$} & \multicolumn{2}{|c|}{$C L=50.0 \mathrm{mg} / \mathrm{L}$} \\
\hline $\begin{array}{c}\text { Reaction } \\
\text { time } \\
\text { (hours) }\end{array}$ & pH & $\begin{array}{c}\text { Reaction } \\
\text { time } \\
\text { (hours) }\end{array}$ & pH & $\begin{array}{c}\text { Reaction } \\
\text { time } \\
\text { (hours) }\end{array}$ & pH \\
\hline \multicolumn{6}{|c|}{ Initial $\mathrm{pH}=\mathbf{5 . 5 0}$} \\
\hline 0.00 & 5.50 & 0.00 & 5.50 & 0.00 & 5.50 \\
\hline 1.42 & 6.80 & 1.30 & 6.93 & 1.18 & 7.42 \\
\hline 24.28 & 6.82 & 24.17 & 6.92 & 24.05 & 7.32 \\
\hline 47.50 & 6.91 & 47.38 & 6.93 & 47.27 & 7.36 \\
\hline 76.25 & 7.05 & 76.05 & 6.96 & 75.85 & 7.41 \\
\hline 124.7 & 7.31 & 124.5 & 7.07 & 124.3 & 7.44 \\
\hline 170.4 & 7.51 & 170.2 & 7.14 & 170.0 & 7.60 \\
\hline \multicolumn{6}{|c|}{ Initial $\mathrm{pH}=\mathbf{7 . 5 0}$} \\
\hline .00 & 7.50 & .00 & 7.50 & .00 & 7.50 \\
\hline 2.80 & 8.41 & 2.53 & 8.56 & 2.73 & 8.81 \\
\hline 25.67 & 8.29 & 25.40 & 8.44 & 25.60 & 8.69 \\
\hline 48.88 & 8.25 & 48.62 & 8.40 & 48.82 & 8.66 \\
\hline 77.35 & 8.26 & 77.02 & 8.39 & 77.13 & 8.63 \\
\hline 125.8 & 8.25 & 125.5 & 8.36 & 125.6 & 8.59 \\
\hline 171.5 & 8.27 & 171.2 & 8.37 & 171.3 & 8.56 \\
\hline \multicolumn{6}{|c|}{ Initial $\mathbf{p H}=\mathbf{1 0 . 0 0}$} \\
\hline .00 & 10.00 & .00 & 10.00 & .00 & 10.00 \\
\hline .75 & 9.98 & .58 & 10.06 & .48 & 10.10 \\
\hline 23.63 & 9.74 & 23.47 & 9.82 & 23.37 & 9.86 \\
\hline 46.83 & 9.64 & 46.67 & 9.74 & 46.57 & 9.79 \\
\hline 75.00 & 9.60 & 74.83 & 9.71 & 74.73 & 9.77 \\
\hline 123.6 & 9.56 & 123.3 & 9.67 & 123.1 & 9.75 \\
\hline 169.3 & 9.56 & 169.0 & 9.68 & 168.9 & 9.73 \\
\hline
\end{tabular}


Table 40.--Variation of $\mathrm{pH}$ with reaction time for different combinations of initial $\mathrm{pH}$ and initial free-chiorine concentration, Mississippi River at Baton Rouge, La., March 26, 1992

[CL, initial free-chlorine concentration; $\mathrm{mg} / \mathrm{L}$, milligram per liter]

\begin{tabular}{|c|c|c|c|c|c|}
\hline \multicolumn{2}{|c|}{$C L=15.0 \mathrm{mg} / \mathrm{L}$} & \multicolumn{2}{|c|}{$C L=30.0 \mathrm{mg} / \mathrm{L}$} & \multicolumn{2}{|c|}{$C L=50.0 \mathrm{mg} / \mathrm{L}$} \\
\hline $\begin{array}{l}\text { Reaction } \\
\text { time } \\
\text { (hours) }\end{array}$ & pH & $\begin{array}{c}\text { Reaction } \\
\text { time } \\
\text { (hours) }\end{array}$ & pH & $\begin{array}{c}\text { Reaction } \\
\text { time } \\
\text { (hours) }\end{array}$ & pH \\
\hline \multicolumn{6}{|c|}{ Initial pH $=\mathbf{5 . 5 0}$} \\
\hline 0.00 & 5.50 & 0.00 & 5.50 & 0.00 & 5.50 \\
\hline 1.42 & 6.75 & 1.32 & 6.95 & 1.23 & 7.38 \\
\hline 54.58 & 6.77 & 54.40 & 7.08 & 54.23 & 7.33 \\
\hline 77.38 & 6.79 & 77.22 & 7.11 & 77.05 & 7.39 \\
\hline 125.5 & 6.84 & 125.4 & 7.12 & 125.2 & 7.47 \\
\hline 170.3 & 6.91 & 170.2 & 7.17 & 170.0 & 7.59 \\
\hline \multicolumn{6}{|c|}{ Initial pH $=\mathbf{7 . 5 0}$} \\
\hline .00 & 7.50 & .00 & 7.50 & .00 & 7.50 \\
\hline 2.30 & 8.58 & 2.30 & 8.58 & 2.52 & 8.82 \\
\hline 55.13 & 8.39 & 55.13 & 8.39 & 55.28 & 8.67 \\
\hline 77.93 & 8.36 & 77.93 & 8.36 & 78.08 & 8.65 \\
\hline 126.1 & 8.33 & 126.1 & 8.33 & 126.2 & 8.61 \\
\hline 170.9 & 8.29 & 170.9 & 8.29 & 171.0 & 8.59 \\
\hline \multicolumn{6}{|c|}{ Initial pH $=10.00$} \\
\hline .00 & 10.00 & .00 & 10.00 & .00 & 10.00 \\
\hline .83 & 10.04 & .73 & 10.07 & .63 & 10.09 \\
\hline 53.53 & 9.70 & 53.37 & 9.72 & 53.20 & 9.79 \\
\hline 76.33 & 9.67 & 76.17 & 9.71 & 76.00 & 9.75 \\
\hline 124.5 & 9.62 & 124.3 & 9.68 & 124.1 & 9.72 \\
\hline 169.3 & 9.59 & 169.1 & 9.66 & 169.0 & 9.70 \\
\hline
\end{tabular}


Tabie 41.-Variation of $\mathrm{pH}$ with reaction time for different combinations of initial $\mathrm{pH}$ and initiai free-chiorine concentration, New Orieans, La., March 25, 1992

[CL, initial free-chlorine concentration; $\mathrm{mg} / \mathrm{L}$, milligram per liter]

\begin{tabular}{|c|c|c|c|c|c|}
\hline \multicolumn{2}{|c|}{$C L=15.0 \mathrm{mg} / \mathrm{L}$} & \multicolumn{2}{|c|}{$\mathrm{CL}=30.0 \mathrm{mg} / \mathrm{L}$} & \multicolumn{2}{|c|}{$\mathrm{CL}=50.0 \mathrm{mg} / \mathrm{L}$} \\
\hline $\begin{array}{c}\text { Reaction } \\
\text { time } \\
\text { (hours) }\end{array}$ & pH & $\begin{array}{c}\text { Reaction } \\
\text { time } \\
\text { (hours) }\end{array}$ & pH & $\begin{array}{c}\text { Reaction } \\
\text { time } \\
\text { (hours) }\end{array}$ & pH \\
\hline \multicolumn{6}{|c|}{ Initial pH $=\mathbf{5 . 5 0}$} \\
\hline 0.00 & 5.50 & 0.00 & 5.50 & 0.00 & 5.50 \\
\hline 1.42 & 6.70 & 1.30 & 6.88 & 1.20 & 7.31 \\
\hline 22.70 & 6.71 & 22.52 & 6.82 & 22.35 & 7.34 \\
\hline 46.35 & 6.80 & 46.17 & 6.87 & 46.00 & 7.47 \\
\hline 71.78 & 6.95 & 71.60 & 6.90 & 71.43 & 7.59 \\
\hline 120.0 & 7.04 & 119.8 & 6.96 & 119.6 & 7.76 \\
\hline 170.3 & 7.16 & 170.1 & 7.09 & 170.0 & 7.90 \\
\hline \multicolumn{6}{|c|}{ Initial $\mathrm{pH}=7.50$} \\
\hline .00 & 7.50 & .00 & 7.50 & .00 & 7.50 \\
\hline 2.72 & 8.38 & 2.42 & 8.55 & 2.63 & 8.79 \\
\hline 23.75 & 8.00 & 23.38 & 8.45 & 23.53 & 8.71 \\
\hline 47.42 & 8.28 & 47.05 & 8.43 & 47.20 & 8.70 \\
\hline 72.85 & 8.26 & 72.48 & 8.40 & 72.63 & 8.70 \\
\hline 121.0 & 8.22 & 120.6 & 8.35 & 120.8 & 8.64 \\
\hline 171.4 & 8.22 & 171.0 & 8.35 & 171.2 & 8.62 \\
\hline \multicolumn{6}{|c|}{ Initial pH = 10.00} \\
\hline .00 & 10.00 & .00 & 10.00 & .00 & 10.00 \\
\hline .82 & 10.03 & .68 & 10.08 & .60 & 10.11 \\
\hline 21.65 & 9.91 & 21.45 & 9.89 & 21.30 & 9.96 \\
\hline 45.32 & 9.82 & 45.12 & 9.81 & 44.97 & 9.88 \\
\hline 70.75 & 9.77 & 70.55 & 9.77 & 70.40 & 9.83 \\
\hline 118.9 & 9.69 & 118.7 & 9.71 & 118.5 & 9.78 \\
\hline 169.2 & 9.67 & 169.0 & 9.67 & 168.9 & 9.74 \\
\hline
\end{tabular}




\section{Values of $\mathrm{pH}$ at the End of the Trihalomethane Formation-Potential Experiments}

Values of $\mathrm{pH}$ at the end of the trihalomethane formation-potential experiments for each of the different combinations of initial $\mathrm{pH}$ and initial free-chlorine concentration are listed in table 42. These $\mathrm{pH}$ values differ somewhat from the $\mathrm{pH}$ values in tables $28-41$ because they were determined on samples that had been quenched with a sodium thiosulfate solution and extracted with pentane, followed by evaporation of the pentane. Most of the small differences are believed to be from the addition of the sodium thiosulfate, which generally increased the $\mathrm{pH}$ values of the 5.50 and 7.50 samples and had little or no effect on the $\mathrm{pH}$ values of the 10.00 samples.

\section{Dissolved Organic-Carbon and Bromide Concentrations and Specific Conductances}

DOC and bromide concentrations and specific conductances for each of the water samples are listed in table 43. Replicate analyses were done for DOC for all samples except the Davenport sample. As indicated previously, three bottles of this water sample froze during storage, and formation-potential experiments were done for both the regular water sample and for the melt water recovered from the thawed frozen sample. DOC concentrations are given in table 43 for the regular water sample and for the thawed water; however, only one analysis was possible because of an insufficient quantity of water. Coefficients of variation of the replicate analyses for the other 13 samples ranged from \pm 0.00 to \pm 3.45 and averaged \pm 0.84 percent. Bromide concentrations ranged from less than the detection limit of $0.005 \mathrm{mg} / \mathrm{L}$ for the Quincy, St. Louis, and Ohio River water samples to $0.040 \mathrm{mg} / \mathrm{L}$ for the Missouri River water sample. An interference problem prevented the determination of the bromide concentrations for the Minneapolis, Dubuque, Davenport, and Cairo water samples.

\section{Ultraviolet Absorbances}

Ultraviolet absorbances of the natural water samples and the water samples adjusted to $\mathrm{pH}$ values of $5.50,7.50$, and 10.00 were measured at $254,280,330$, and $400 \mathrm{~nm}$, and the results are listed in table 44. Because three bottles of the Davenport water sample froze during storage, formationpotential experiments were done both for the regular water sample and for the melt water recovered from the thawed frozen sample. Ultraviolet absorbances are listed in table 44 for both of these water samples. The $\mathrm{pH}$ value of the natural water sample for each sample location is the average of the $\mathrm{pH}$ values for the three volumes of water used for the formation-potential experiments and the one volume used for the preparation of the THM standards. Slight precipitates were observed in the Minneapolis, LaCrosse, Dubuque, Davenport (regular and thawed), Quincy, Missouri River, St. Louis, and Cairo water samples for the $\mathrm{pH}=10.00$ condition. The extent to which the UV absorbances of these samples were affected by this precipitate is unknown. 
Table 42.-Values of $\mathrm{pH}$ at the end of the trihalomethane formation-potential experiments

$[\mathrm{CL}$, initial free-chlorine concentration; $\mathrm{mg} / \mathrm{L}$, milligram per liter; NS, no sample because of insufficient water]

\begin{tabular}{|c|c|c|c|c|}
\hline \multirow[t]{2}{*}{ General location } & \multirow{2}{*}{$\begin{array}{c}\text { Initiai } \\
\text { pH }\end{array}$} & \multicolumn{3}{|c|}{$\begin{array}{c}\mathrm{Cl} \\
(\mathrm{mg} / \mathrm{L})\end{array}$} \\
\hline & & 15.0 & 30.0 & 50.0 \\
\hline \multirow[t]{3}{*}{ Minneapolis, Minn. } & 5.50 & 6.68 & 6.84 & 7.08 \\
\hline & 7.50 & 8.12 & 8.29 & 8.64 \\
\hline & 10.00 & 9.52 & 9.62 & 9.69 \\
\hline \multirow[t]{3}{*}{ LaCrosse, Wis. } & 5.50 & 6.86 & 6.93 & 7.11 \\
\hline & 7.50 & 8.09 & 8.18 & 8.65 \\
\hline & 10.00 & 9.23 & 9.43 & 9.49 \\
\hline \multirow[t]{3}{*}{ Dubuque, Iowa } & 5.50 & 7.03 & 7.14 & 7.52 \\
\hline & 7.50 & 8.19 & 8.34 & 8.81 \\
\hline & 10.00 & 9.33 & 9.49 & 9.60 \\
\hline \multirow[t]{3}{*}{ Davenport, Iowa (regular sample) } & 5.50 & NS & 7.04 & 7.32 \\
\hline & 7.50 & NS & 8.42 & 8.81 \\
\hline & 10.00 & 9.38 & 9.48 & 9.62 \\
\hline \multirow[t]{3}{*}{ Davenport, Iowa (thawed sample) } & 5.50 & NS & 6.98 & 7.26 \\
\hline & 7.50 & NS & 8.42 & 8.83 \\
\hline & 10.00 & NS & 9.53 & 9.63 \\
\hline \multirow[t]{3}{*}{ Quincy, Ill. } & 5.50 & 7.15 & 7.26 & 7.52 \\
\hline & 7.50 & 8.32 & 8.33 & 8.83 \\
\hline & 10.00 & 9.45 & 9.56 & 9.66 \\
\hline \multirow[t]{3}{*}{ Missouri River near confluence with Mississippi River } & 5.50 & 6.97 & 7.10 & 7.41 \\
\hline & 7.50 & 8.12 & 8.32 & 8.66 \\
\hline & 10.00 & 9.21 & 9.37 & 9.65 \\
\hline \multirow[t]{3}{*}{ St. Louis, Mo. } & 5.50 & 7.19 & 7.33 & 7.75 \\
\hline & 7.50 & 8.47 & 8.50 & 8.91 \\
\hline & 10.00 & 9.48 & 9.59 & 9.68 \\
\hline \multirow[t]{3}{*}{ Cairo, Ill. } & 5.50 & 6.82 & 6.96 & 7.39 \\
\hline & 7.50 & 8.29 & 8.54 & 8.97 \\
\hline & 10.00 & 9.50 & 9.61 & 9.74 \\
\hline \multirow[t]{3}{*}{ Ohio River near confluence with Mississippi River } & 5.50 & 7.17 & 7.54 & 8.37 \\
\hline & 7.50 & 8.39 & 8.96 & 9.26 \\
\hline & 10.00 & 9.44 & 9.58 & 9.71 \\
\hline
\end{tabular}


Table 42.-Values of $\mathrm{pH}$ at the end of the trihalomethane formation-potential experiments -Continued

\begin{tabular}{|c|c|c|c|c|}
\hline \multirow{2}{*}{ General location } & \multirow{2}{*}{$\begin{array}{c}\text { Initiai } \\
\text { pH }\end{array}$} & \multicolumn{3}{|c|}{$\underset{(m g / L)}{C L}$} \\
\hline & & 15.0 & 30.0 & 50.0 \\
\hline \multirow[t]{3}{*}{ Memphis, Tenn. } & 5.50 & 7.13 & 7.28 & 7.82 \\
\hline & 7.50 & 8.36 & 8.76 & 9.19 \\
\hline & 10.00 & 9.48 & 9.66 & 9.78 \\
\hline \multirow[t]{3}{*}{ Greenville, Miss. } & 5.50 & 6.67 & 6.99 & 7.50 \\
\hline & 7.50 & 8.22 & 8.66 & 9.03 \\
\hline & 10.00 & 9.50 & 9.66 & 9.75 \\
\hline \multirow[t]{3}{*}{ Natchez, Miss. } & 5.50 & 7.07 & 7.28 & 8.00 \\
\hline & 7.50 & 8.31 & 8.72 & 9.04 \\
\hline & 10.00 & 9.40 & 9.65 & 9.76 \\
\hline \multirow[t]{3}{*}{ Baton Rouge, La. } & 5.50 & 7.02 & 7.31 & 8.12 \\
\hline & 7.50 & 8.50 & 8.87 & 9.23 \\
\hline & 10.00 & 9.63 & 9.74 & 9.85 \\
\hline \multirow[t]{3}{*}{ New Orleans, La. } & 5.50 & 6.99 & 7.24 & 7.87 \\
\hline & 7.50 & 8.33 & 8.74 & 9.05 \\
\hline & 10.00 & 9.41 & 9.60 & 9.76 \\
\hline
\end{tabular}


Table 43.-Dissolved organic-carbon and bromide concentrations and specific conductances of the water samples

[DOC, dissolved organic carbon; Br, bromide; Rep 1, replicate number 1; Rep 2, replicate number $2 ; \mathrm{mg} / \mathrm{L}$, milligram per liter; $\mu \mathrm{S} / \mathrm{cm}$, microsiemens per centimeter at 25 degrees Celsius; ND, not detected; IF, interference problem; NS, no sample because of insufficient water]

\begin{tabular}{|c|c|c|c|c|}
\hline \multirow{3}{*}{ General location } & \multicolumn{3}{|c|}{ Concentration } & \multirow{3}{*}{$\begin{array}{c}\text { Specific } \\
\text { conductance } \\
(\mu \mathrm{S} / \mathrm{cm})\end{array}$} \\
\hline & \multicolumn{2}{|c|}{ DOC } & \multirow{2}{*}{$\begin{array}{c}\text { Br } \\
(\mathrm{mg} / \mathrm{L})\end{array}$} & \\
\hline & $\begin{array}{l}\text { Rep 1 } \\
\text { (mg/L) }\end{array}$ & $\begin{array}{l}\text { Rep } 2 \\
\text { (mg/L) }\end{array}$ & & \\
\hline Minneapolis, Minn. & 9.3 & 9.4 & IF & 402 \\
\hline LaCrosse, Wis. & 7.2 & 7.3 & 0.010 & 495 \\
\hline Dubuque, Iowa & 6.9 & 6.8 & IF & 407 \\
\hline $\begin{array}{l}\text { Davenport, lowa } \\
\text { (regular sample) }\end{array}$ & 6.4 & NS & IF & 422 \\
\hline $\begin{array}{l}\text { Davenport, Iowa } \\
\text { (thawed sample) }\end{array}$ & 6.0 & NS & IF & 388 \\
\hline Quincy, Ill. & 4.7 & 4.7 & ND & 496 \\
\hline Missouri River $^{1}$ & 4.3 & 4.2 & .040 & 530 \\
\hline St. Louis, Mo. & 4.2 & 4.5 & ND & 495 \\
\hline Cairo, Ill. & 4.8 & 4.7 & IF & 478 \\
\hline Ohio River ${ }^{1}$ & 2.2 & 2.2 & ND & 275 \\
\hline Memphis, Tenn. & 3.5 & 3.5 & .011 & 348 \\
\hline Greenville, Miss. & 3.2 & 3.3 & .010 & 340 \\
\hline Natchez, Miss. & 2.9 & 2.8 & .016 & 312 \\
\hline Baton Rouge, La. & 3.0 & 3.0 & .014 & 309 \\
\hline New Orleans, La. & 2.8 & 2.8 & .030 & 333 \\
\hline
\end{tabular}

${ }^{1}$ Sampling site 1.6 kilometers upstream from confluence with Mississippi River. 
Table 44.-Ultraviolet absorbances of the water samples

[nm, nanometer]

\begin{tabular}{|c|c|c|c|c|c|}
\hline \multirow{2}{*}{ General location } & \multirow{2}{*}{$\mathrm{pH}$} & \multicolumn{4}{|c|}{$\begin{array}{l}\text { Ultraviolet absorbance, in absorbance units, } \\
\text { at wavelengths }\end{array}$} \\
\hline & & $\begin{array}{l}254 \\
(\mathrm{~nm})\end{array}$ & $\begin{array}{l}280 \\
(\mathrm{~nm})\end{array}$ & $\begin{array}{l}330 \\
(\mathrm{~nm})\end{array}$ & $\begin{array}{l}400 \\
(n m)\end{array}$ \\
\hline \multirow[t]{4}{*}{ Minneapolis, Minn. } & 8.02 & 1.356 & 0.981 & 0.424 & 0.122 \\
\hline & 5.50 & 1.344 & .970 & .405 & .109 \\
\hline & 7.50 & 1.341 & .968 & .414 & .155 \\
\hline & 10.00 & 1.289 & .948 & .459 & .218 \\
\hline \multirow[t]{4}{*}{ LaCrosse, Wis. } & 8.04 & 1.101 & .806 & .359 & .107 \\
\hline & 5.50 & 1.076 & .786 & .336 & .092 \\
\hline & 7.50 & 1.111 & .815 & .360 & .104 \\
\hline & 10.00 & 1.028 & .809 & .413 & .203 \\
\hline \multirow[t]{4}{*}{ Dubuque, Iowa } & 8.06 & 1.084 & .811 & .371 & .121 \\
\hline & 5.50 & 1.026 & .759 & .337 & .099 \\
\hline & 7.50 & 1.031 & .764 & .346 & .105 \\
\hline & 10.00 & .942 & .700 & .320 & . 103 \\
\hline \multirow{3}{*}{$\begin{array}{l}\text { Davenport, Iowa } \\
\text { (regular sample) }\end{array}$} & $\begin{array}{l}8.10 \\
5.50\end{array}$ & $\begin{array}{l}.954 \\
.946\end{array}$ & $\begin{array}{l}.703 \\
.696\end{array}$ & .313 & $\begin{array}{l}.093 \\
.086\end{array}$ \\
\hline & 7.50 & .974 & .721 & .319 & .095 \\
\hline & 10.00 & .878 & .636 & .279 & .077 \\
\hline \multirow{4}{*}{$\begin{array}{l}\text { Davenport, Iowa } \\
\text { (thawed sample) }\end{array}$} & 8.14 & .933 & .692 & .312 & .097 \\
\hline & 5.50 & .926 & .687 & .302 & .090 \\
\hline & 7.50 & .929 & .690 & .308 & .096 \\
\hline & 10.00 & .928 & .656 & .299 & .101 \\
\hline \multirow[t]{4}{*}{ Quincy, Ill. } & 8.16 & .723 & .529 & .228 & .066 \\
\hline & 5.50 & .723 & .528 & .221 & .058 \\
\hline & 7.50 & .735 & .541 & .231 & .066 \\
\hline & 10.00 & .693 & .534 & .274 & .139 \\
\hline \multirow{4}{*}{$\begin{array}{l}\text { Missouri River near conflu- } \\
\text { ence with Mississippi River }\end{array}$} & 7.95 & .610 & .451 & & .059 \\
\hline & 5.50 & .602 & .441 & 191 & .054 \\
\hline & 7.50 & .605 & .445 & 198 & .059 \\
\hline & 10.00 & .551 & .398 & .174 & .050 \\
\hline
\end{tabular}


Table 44.-Ultraviolet absorbances of the water samples -Continued

\begin{tabular}{|c|c|c|c|c|c|}
\hline \multirow{2}{*}{ Generai location } & \multirow{2}{*}{ pH } & \multicolumn{4}{|c|}{$\begin{array}{l}\text { Uitraviolet absorbance, in absorbance units, } \\
\text { at waveiengths }\end{array}$} \\
\hline & & $\begin{array}{l}254 \\
(\mathrm{~nm})\end{array}$ & $\begin{array}{l}280 \\
(\mathrm{~nm})\end{array}$ & $\begin{array}{c}330 \\
(\mathrm{~nm})\end{array}$ & $\begin{array}{c}400 \\
(\mathrm{~nm})\end{array}$ \\
\hline \multirow[t]{4}{*}{ St. Louis, Mo. } & 7.98 & .668 & .491 & .213 & .062 \\
\hline & 5.50 & .671 & .491 & .208 & .056 \\
\hline & 7.50 & .679 & .498 & .216 & .063 \\
\hline & 10.00 & .599 & .437 & .191 & .059 \\
\hline \multirow[t]{4}{*}{ Cairo, Ill. } & 7.98 & .709 & .519 & .224 & .064 \\
\hline & 5.50 & .703 & .513 & .214 & .054 \\
\hline & 7.50 & .707 & .519 & .222 & .064 \\
\hline & 10.00 & .673 & .494 & .219 & .071 \\
\hline \multirow{4}{*}{$\begin{array}{l}\text { Ohio River near confluence } \\
\text { with Mississippi River }\end{array}$} & 7.76 & .349 & .262 & .114 & .034 \\
\hline & 5.50 & .345 & .256 & .111 & .031 \\
\hline & 7.50 & .345 & .258 & .116 & .033 \\
\hline & 10.00 & .357 & .270 & .124 & .041 \\
\hline \multirow[t]{4}{*}{ Memphis, Tenn. } & 7.80 & .506 & .378 & .168 & .050 \\
\hline & 5.50 & .505 & .372 & .162 & .046 \\
\hline & 7.50 & .507 & .379 & .168 & .049 \\
\hline & 10.00 & .515 & .386 & .180 & .058 \\
\hline \multirow[t]{4}{*}{ Greenville, Miss. } & 7.82 & .481 & .356 & .158 & .048 \\
\hline & 5.50 & .481 & .354 & .152 & .043 \\
\hline & 7.50 & .480 & .355 & .156 & .046 \\
\hline & 10.00 & .493 & .366 & .169 & .055 \\
\hline \multirow[t]{4}{*}{ Natchez, Miss. } & 7.85 & .476 & .355 & .157 & .049 \\
\hline & 5.50 & .476 & .353 & .152 & .044 \\
\hline & 7.50 & .479 & .358 & .157 & .049 \\
\hline & 10.00 & .496 & .372 & .173 & .059 \\
\hline \multirow[t]{4}{*}{ Baton Rouge, La. } & 7.82 & .459 & .343 & .153 & .044 \\
\hline & 5.50 & .461 & .343 & .150 & .041 \\
\hline & 7.50 & .464 & .349 & .156 & .046 \\
\hline & 10.00 & .474 & .357 & .165 & .053 \\
\hline
\end{tabular}


Table 44.-Ultraviolet absorbances of the water samples -Continued

\begin{tabular}{lccccc}
\hline \multirow{2}{*}{ General location } & pH & \multicolumn{4}{c}{$\begin{array}{c}\text { Ultraviolet absorbance, in absorbance units, } \\
\text { at wavelengths }\end{array}$} \\
\cline { 3 - 6 } & & $\mathbf{2 5 4}$ & $\mathbf{2 8 0}$ & $\mathbf{3 3 0}$ & $\mathbf{4 0 0}$ \\
& & $\mathbf{( n m )}$ & $\mathbf{( n m )}$ & $\mathbf{( n m )}$ & $\mathbf{( n m )}$ \\
\hline New Orleans, La. & 7.78 & .409 & .301 & .131 & .038 \\
& 5.50 & .407 & .297 & .126 & .034 \\
& 7.50 & .411 & .303 & .132 & .039 \\
& 10.00 & .415 & .308 & .139 & .043 \\
\hline
\end{tabular}




\section{Error Analysis}

The coefficient of variation normalized with respect to the mean concentration and expressed as a percentage was used to indicate reproducibility between duplicate samples. This coefficient of variation was computed from

where

$$
C_{v}= \pm\left\{\left[C_{1}^{2}+C_{2}{ }^{2}-\left(C_{1}+C_{2}\right)^{2} / 2\right] / 2\right\}^{0.5} 100 / C_{12}
$$

$\mathrm{C}_{\mathrm{v}}$ is the coefficient of variation;

$\mathrm{C}_{1}$ is concentration number 1 ;

$\mathrm{C}_{2}$ is concentration number 2 ; and

$\mathrm{C}_{12}$ is the mean of concentrations 1 and 2 .

Values of the coefficients of variation for the analysis of duplicate THM samples and NPTOX samples are summarized in table 45. Minimum, maximum, and mean values of the coefficient of variation together with the number of pairs of values in each comparison are listed.

Table 45.-Minimum, maximum, and mean values of the coefficient of variation for analysis of duplicate trihalomethane and nonpurgeable total organic-halide samples

[THM, trihalomethane; NPTOX, nonpurgeable total organic halide]

\begin{tabular}{lcccc}
\hline \multirow{2}{*}{ Sampie type } & $\begin{array}{c}\text { Number of } \\
\text { pairs }\end{array}$ & \multicolumn{3}{c}{ Coefficient of variation, in percent } \\
\cline { 3 - 5 } & & Minimum & Maximum & Mean \\
\hline THM & 130 & \pm 0.00 & \pm 4.75 & \pm 0.79 \\
NPTOX & 130 & \pm .00 & \pm 6.45 & \pm 1.83 \\
\hline
\end{tabular}




\section{REFERENCES CITED}

Bellar, T.A., Lichtenberg, J.J., and Kroner, R.C., 1974, The occurrence of organohalides in chlorinated drinking waters: Journal of American Water Works Association, v. 66, no. 12, p. 703-706.

Buffle, J., Deladoey, P., and Haerdi, W., 1978, The use of ultrafiltration for the separation and fractionation of organic ligands in fresh waters: Analytica Chimica Acta, v. 101, no. 2, p. 339-357.

Ceraso, Jane, 1987, Development of a surrogate parameter to determine the trihalomethane formation potential of two reservoirs: Journal of New England Water Works Association, v. 101, no. 4 , p. $382-400$.

Chadick, P.A., and Amy, G.L., 1987, Coagulation and adsorption of humic substances-An analysis of surrogate parameters for predicting effects of trihalomethane formation potential: Environmental Technology Letters, v. 8, p. 261-268.

Edzwald, J.K., Becker, W.C.; and Wattier, K.L., 1985, Surrogate parameters for monitoring organic matter and THM precursors: Journal of American Water Works Association, v. 77, no. 4, p. 122-132.

Federal Register, 1979, Analysis of trihalomethanes in drinking water, v. 44, no. 231, p. $68672-68690$.

Fishman, M.J., and Friedman, L.C., 1989, Methods for determination of inorganic substances in water and fluvial sediments: U.S. Geological Survey Techniques of Water-Resources Investigations Report, book 5, chap. A1, 545 p.

Fleischacker, S.J., and Randtke, S.J., 1983, Formation of organic chlorine in public water supplies: Journal of American Water Works Association, v. 75, no. 3, p. 132-138.

Johnson, J.D., and Jensen, J.N., 1986, THM and TOX formation-Routes, rates, and precursors: Journal of American Water Works Association, v. 78, no. 4, p. 156-162.

Moody, J.A., in press, Chapter 1--Introduction, in Moody, J.A., editor, Chemical data for water samples collected druing four upriver cruises on the Mississippi River between New Orleans, Louisiana, and Minneapolis, Minnesota, May 1990-April 1992: U.S. Geological Survey OpenFile Report 94-523.

Moore, T.R., 1987, Assessment of a simple spectrophotometric method for the determination of dissolved organic carbon in freshwaters: New Zealand Journal of Marine and Freshwater Research, v. 21, no. 4, p. 585-589.

Oliver, B.G., and Thurman, E.M., 1981, Influence of aquatic humic substances on trihalomethane potential, in Proceedings, Fourth Conference on Water Chlorination, October 18-23, 1981, Environmental Impact and Health Effects: Ann Arbor, Mich., Ann Arbor Science Publishers, p. 231-241.

Rook, J.J., 1974, Formation of haloforms during chlorination of natural waters: Water Treatment and Examination, v. 23, no. 2, p. 234-243.

Wershaw, R.L., Fishman, M.J., Grabbe, R.R., and Lowe, L.E., 1987, Methods for the determination of organic substances in water and fluvial sediments: U.S. Geological Survey Techniques of Water-Resources Investigations, book 5, chap. A3, 80 p. 